\title{
THE HOLOMORPHIC DISCRETE SERIES OF AN AFFINE SYMMETRIC SPACE AND REPRESENTATIONS WITH REPRODUCING KERNELS
}

\author{
G. 'OLAFSSON AND B. ØRSTED
}

\begin{abstract}
Consider a semisimple connected Lie group $G$ with an affine symmetric space $X$. We study abstractly the intertwining operators from the discrete series of $X$ into representations with reproducing kernel and, in particular, into the discrete series of $G$; each such is given by a convolution with an analytic function. For $X$ of Hermitian type, we consider the holomorphic discrete series of $X$ and here derive very explicit formulas for the intertwining operators. As a corollary we get a multiplicity one result for the series in question.
\end{abstract}

\section{INTRODUCTION}

The purpose of this work is twofold. The first is to study abstractly the intertwining operators between discrete series representations of an affine symmetric space $X$ and discrete series of the corresponding semisimple group. The second is to obtain very explicit formulas for certain discrete representations on $X$, in effect giving the analogue of Harish-Chandra's original construction of the holomorphic discrete series in the group case. Not surprisingly, these two problems are highly connected. Thus Theorem 5.4 below gives the formula for the lowest $K$-type (the Flensted-Jensen function) on $X$, whereas in Theorem 7.3 essentially the same function gives the intertwining operator. Much of our analysis builds on the framework in [14], but otherwise we have tried to make the paper reasonably self-contained. This is, in particular, the case in $\S 1, \S 2$, and $\S 4$, where we abstract the properties of our intertwining operators with an eye towards later applications.

We shall always let $G$ be a connected, noncompact semisimple Lie group such that the associated Riemannian symmetric space is a bounded symmetric domain $D \subset \mathbb{C}^{n}$. The best known and most interesting discrete series representations of $G$ are the holomorphic discrete series representations. As explained above, one of our aims in this paper is to generalize this construction to affine symmetric spaces of Hermitian type and relate those with other well-known representations.

Received by the editors September 23, 1988 and, in revised form, June 27, 1989.

1980 Mathematics Subject Classification (1985 Revision). Primary 22E46; Secondary 43A85. 
In [14] we did this for the case of a holomorphic character of the universal covering of the complexification of a maximal compact subgroup. One of our main tools in that paper was the analysis of an intertwining operator into the holomorphic discrete series of $G$, and the fact that such an intertwining operator is determined by the reproducing kernel of the representation in its standard realization in the space of holomorphic functions on $D$. It turns out that this connection between the intertwining operators and the reproducing kernel only depends on the existence of a kernel. Hence this idea can be used to analyze other realizations of such representations, e.g. in $\mathbf{L}^{2}$ of an affine symmetric space $X$.

Many interesting representations of $G$ can be realized in $\mathscr{C}^{\infty}$-vector bundle over a homogeneous $G$-space such that the representation has a reproducing kernel, e.g. the ladder representations, the analytic continuation of the holomorphic discrete series, and also-by a construction of Hotta, Wallach, and Wolf-the discrete series in general. In Gutkin [6] one finds a nice abstraction of these examples, namely the concept of elliptic representations. These are realized in the square-integrable kernel of an invariant elliptic operator on a homogeneous Hermitian vector bundle. We collect some simple facts concerning intertwining operators and reproducing kernels in the first section of this paper. By this we get a general formula for the reproducing kernel in term of the "lowest" $K$-type, and an abstract proof of the fact used in [14] that the inverse of an intertwining operator of a reproducing representation, i.e. a representation with a reproducing kernel, into $\mathbf{L}^{2}(X)$, is given by a convolution with a $K$-finite, analytic function. Another application of the ideas in $\S 1$ is a simple proof in $\S 2$ of the reproducing property of the general discrete series in the realization of Hotta. As a corollary of this, we have that every intertwining operator of a discrete series representation of $X$ into a discrete series representation of $G$ is a convolution operator. Given such an imbedding $U$ there exists an analytic function $\phi$ on $X$ with values in the space of the lowest $K$-type of $\pi$ such that (assuming for simplicity that the center of $G$ is finite)

$$
U(g)(y)=\int_{X} g(x) \phi\left(y^{-1} x\right) d x
$$

and if $\pi$ is integrable

$$
T(f)(x)=\int_{D}\left(f(z), \phi\left(z^{-1} x\right)\right)_{V} d z
$$

where $T$ is the inverse of $U$ (see Theorems 3.2 and 4.2). It should be noted that in the literature such representations have been studied, e.g., by H. Schlichtkrull [17] by determining the Langlands-parameters of some of the representations in [4].

In [14] we found an explicit formula for $\phi$ in the case of holomorphic line bundles over the symmetric space. In the general case we can only sayanalogously to the lowest $K$-type of the corresponding representation-that the 
function is given via the Flensted-Jensen-isomorphism and the Poisson transformation of a vector-valued distribution on the boundary of the dual noncompact Riemannian form $X^{r}=G^{d} / K^{d}$ of $X$. But if $X$ is of Hermitian type and the representation is holomorphic, we can give an explicit formula for $\phi$. We turn to this case in the second part of this paper, $\S \S 5-7$. First we associate to some $K$ types $\delta$ a vector-valued, $K$-finite function $\Phi(x)=\delta^{\vee}\left(\gamma^{\#}(x)\right) \nu^{0}$ on $X^{\#}$, the universal covering of $X$, such that the functions $\langle\Phi(\cdot), v\rangle, v \in \mathbf{V}_{\delta}$, generate the lowest $K$-type of an irreducible discrete series representation $\left(\varepsilon_{\delta}, \mathbf{H}_{\delta}\right)$ of $X^{\#}$. Here $\delta^{\vee}$ is the contragredient of $\delta, \gamma^{\#}(x)$ is determined by the $H_{c}$-component of $x^{-1}$ in the decomposition $G \subset H_{c} K_{c} P^{+}$, and $\nu^{0}$ is $K_{c}^{\# \tau}$-invariant.

By using our results from [14] about $\gamma^{\#}$, we also have a formula for $\Phi$ on a maximal flat submanifold $B$ given by products of cosh-factors. We also use the definition of $\gamma^{\#}$ and its relation with the Iwasawa-decomposition on the "dual"-group $G^{d}$ to relate this with the functions of Flensted-Jensen in [3]. In $\S 6$ we then show that in the case $X=(G \times G) / \operatorname{diagonal}(G) \cong G$ our construction gives the holomorphic discrete series of the group. In the last section we then use the results of $\S 1$ and $\S 3$ to show that our representations are equivalent to the (analytic continuation of the) holomorphic discrete series of the group and that the intertwining operators also are determined by $\Phi$. In particular we have a simple proof of the fact that those representations occur with multiplicity one in $\mathbf{L}^{2}(X)$.

It should be remarked here that there is also a construction with quite different methods of the holomorphic discrete series by Matsumoto in [11]. Furthermore those results also hold for $G$ in the Harish-Chandra class. But as this excludes the case where $G$ has infinite center and makes the notation more complicated as we have to take care of both the infinitesimal character and the lowest $K$-type, we do not handle it here and leave it to the reader to make the necessary changes.

The main ideas of the first part of this paper first appeared in [15], whereas the last part is taken from a lecture by the first author given at the University of Göttingen in the spring of 1988 . The authors would like to thank the Department of Mathematics and Computer Science at the University of Odense and the Sonderforschungsbereich 170 in Göttingen for their support, thus making our joint work during the last year possible.

\section{REPRESENTATIONS WITH REPRODUCING KERNEL}

Let $G$ be a locally compact, Hausdorff topological group and $K$ a closed subgroup of $G$. By a representation of $G$ we mean a continuous representation in a locally convex, complete, Hausdorff, topological vector space over $\mathbb{C}$. To ensure that the contragredient representation defined below is also continuous we assume, furthermore, that the representation space is semireflexive. Let $\rho$ be a finite-dimensional unitary representation of $K$ in $\mathbf{V}$. Let $\mathbf{E}$ be a 
topological vector space of continuous functions $f: G \rightarrow \mathbf{V}$, such that $f(x k)=$ $\rho\left(k^{-1}\right) f(x), x \in G, k \in K$, and let $\pi$ be a representation of $G$ in $\mathbf{E}$ given by

$$
[\pi(x) f](y)=m(x, y) l_{x}(f)(y),
$$

where $l$ denotes the usual left translation and $m: G \times G \rightarrow \mathrm{GL}(\mathbf{V})$ is continuous. We will assume that $\mathrm{ev}_{1}: \mathbf{E} \rightarrow \mathbf{V}, f \mapsto f(1)$ is continuous and surjective. As $\mathrm{ev}_{x}=m\left(x^{-1}, 1\right)^{-1} \circ \mathrm{ev}_{1} \circ \pi\left(x^{-1}\right) \mathrm{ev}_{x}$ is continuous for all $x \in G$. Let $x, y, z \in G$ and $k \in K$. As $\pi$ is a representation of $G$, the following holds with $m(x):=m(x, 1)$ :

$$
\begin{gathered}
m(x y, z)=m(x, z) m\left(y, x^{-1} z\right), \quad m(x, y)=m\left(y^{-1}\right)^{-1} m\left(y^{-1} x\right), \\
\rho(k) m(x, y)=m\left(x, y k^{-1}\right) \rho(k), \\
\mathrm{ev}_{x^{-1} y k}=\rho\left(k^{-1}\right) m(x, y)^{-1} \circ \mathrm{ev}_{y} \circ \pi(x) .
\end{gathered}
$$

Lemma 1.1. Define $\delta: K \mapsto \mathrm{GL}(\mathbf{V})$ by $\delta(k):=m(k) \rho(k)$. Then $\delta$ is a representation of $K$ and $\mathrm{ev}_{1} \in \operatorname{Hom}_{K}(\pi, \delta)$.

Proof. This follows immediately from (1.3) and the fact that $\pi$ is a representation.

Let $\mathbf{E}^{*}$ be the dual of $\mathbf{E}$. As $\mathbf{V}$ is finite dimensional we can identify $\operatorname{Hom}(\mathbf{E}, \mathbf{V})$ with $\mathbf{E}^{*} \otimes \mathbf{V}$ via the pairing

$$
\langle f, \phi \otimes v\rangle_{E}:=\langle f, \phi\rangle v, \quad f \in \mathbf{E}, \phi \in \mathbf{E}^{*}, v \in \mathbf{V},
$$

where $\langle\cdot, \cdot\rangle$ is the usual pairing between $\mathbf{E}$ and $\mathbf{E}^{*}$. By this, ev $_{x}$ corresponds to an element $\mathscr{K}_{x} \in \mathbf{E}^{*} \otimes \mathbf{V}$ such that

$$
f(x)=\left\langle f, \mathscr{K}_{x}\right\rangle_{E}, \quad f \in \mathbf{E}, \quad x \in G .
$$

We call $\mathscr{K}$ the reproducing kernel of $\pi$. As usual, we define a representation $\pi^{\vee}$ on $\mathbf{E}^{*}$ by $\pi^{\vee}(a)={ }^{t} \pi\left(a^{-1}\right)$. As $\mathbf{E}$ is assumed semireflexive, $\pi^{\vee}$ is continuous (see [24, p. 224]). Furthermore $\left\langle f,\left(\pi^{\vee} \otimes 1\right)(a) T\right\rangle_{E}=\left\langle\pi\left(a^{-1}\right) f, T\right\rangle_{E}$ for all $T \in \mathbf{E}^{*} \otimes \mathbf{V}$. Now (1.3) reads

$$
\mathscr{K}_{x y k}=\pi^{\vee}(x) \otimes \rho\left(k^{-1}\right) m\left(x^{-1}, y\right)^{-1} \mathscr{K}_{y} .
$$

Theorem 1.2. Let $v_{1}, \ldots, v_{n}$ be a basis of $\mathbf{V}$ and let $\nu_{1}, \ldots, \nu_{n}$ be the dual basis of $\mathbf{V}^{*}$, i.e. $\left\langle v_{i}, \nu_{j}\right\rangle=\delta_{i j}$. Define $\phi_{i} \in \mathbf{E}^{*}$ by $\phi_{i}:={ }^{t}\left(\mathbf{e v}_{1}\right)\left(\nu_{i}\right)$. Then

(1) $\phi_{i}$ is nonzero and in $\mathbf{E}^{*}\left(\delta^{\vee}\right)$.

(2) $\mathscr{K}_{1} \in \mathbf{E}^{*}\left(\delta^{\vee}\right) \otimes \mathbf{V}$ and $\left(\pi^{\vee}(k) \otimes 1\right) \mathscr{K}_{1}=\left(1 \otimes \delta\left(k^{-1}\right)\right) \mathscr{K}_{1}$.

(3) $\mathscr{K}_{x}=\sum_{i=1}^{n} \pi^{\vee}(x) \phi_{i} \otimes m\left(x^{-1}, 1\right)^{-1} v_{i}$.

In particular, if $G$ is a semisimple Lie group, $K$ is a maximal compact subgroup and $\mathbf{E}^{*}$ is $Z(\mathfrak{g})$-finite, then $\mathscr{K}_{x} \in \mathbf{E}_{\omega}^{*} \otimes \mathbf{V}$, where $\mathbf{E}_{\omega}^{*}$ is the space of analytic vectors of $\mathbf{E}^{*}$. 
Proof. As $\mathrm{ev}_{1}$ is a surjective $(\pi, \delta)$-homomorphism as a representation of $K$, it follows that ${ }^{t}\left(\mathrm{ev}_{1}\right): \mathbf{V}^{*} \rightarrow \mathbf{E}^{*}$ is an injective $\left(\delta^{\vee}, \pi^{\vee}\right)$-homomorphism. Thus $\phi_{i} \neq 0$ and in $\mathbf{E}^{*}\left(\delta^{\vee}\right)$. As

$$
\left\langle f,\left(\pi^{\vee}(k) \otimes 1\right) \mathscr{K}_{1}\right\rangle=\left\langle\pi\left(k^{-1}\right) f, \mathscr{K}_{1}\right\rangle=m\left(k^{-1}, 1\right) \rho\left(k^{-1}\right) f(1),
$$

$f \in \mathbf{E}$, Part (2) follows. By (1.3) ${ }^{\prime}$ we have to show that $\mathscr{K}_{1}=\sum \phi_{i} \otimes v_{i}$. But this follows from

$$
f(1)=\sum_{j=1}^{n}\left\langle f(1), \nu_{j}\right\rangle v_{j}=\sum_{j=1}^{n}\left\langle f,{ }^{t}\left(\mathrm{ev}_{1}\right)\left(\nu_{j}\right)\right\rangle v_{j}=\left\langle f, \sum_{j=1}^{n} \phi_{j} \otimes v_{j}\right\rangle_{E} .
$$

If $G, K$, and $\mathbf{E}^{*}$ are as in the last part of the theorem, then apply the first part and consider $\mathscr{K}_{1}=\sum \phi_{i} \otimes v_{i}$ which is $K$ - and $Z(\mathfrak{g})$-finite for the representation $\pi^{\vee} \otimes 1$. By standard arguments (see [21, p. 312ff]) the claim follows.

Assume now that $\mathbf{E}$ is a Hilbert space and that $\pi$ is unitary. We denote the inner product in a Hilbert space $\mathbf{F}$ by $(\cdot \mid \cdot)_{F}$. Let $j_{V}: \mathbf{V} \rightarrow \mathbf{V}$ be a conjugation of $\mathbf{V}$ and identify $\mathbf{V}^{*}$ with $\mathbf{V}$ via $j_{V}$. For $f, g \in \mathbf{E}$ define $j_{E}(f)(x):=j_{V}(f(x))$ and $\left\langle g, j_{E}(f)\right\rangle:=(g \mid f)$. Then $j_{E}: \mathbf{E} \rightarrow \mathbf{E}^{*}$ is a conjugate-linear isomorphism. Thus $\mathbf{E}^{*}$ is a space of functions from $G$ into $\mathbf{V}^{*}$ such that $\phi(x k)=\rho^{\vee}\left(k^{-1}\right) \phi(x)$ for all $\phi \in \mathbf{E}^{*}$, and $\mathscr{K}$ is identified with a function $\mathscr{K}: G \times G \rightarrow \mathbf{V}^{*} \otimes \mathbf{V}=\operatorname{End}(\mathbf{V})$. As $\pi$ is unitary it follows that $\pi^{\vee}(x)\left(j_{E} g\right)=\bar{m}(x, \cdot) l_{x} j_{E} g$, where $\bar{m}(x, y)=j_{V} m(x, y) j_{V} \in \mathrm{GL}\left(\mathbf{V}^{*}\right)$. Define $\Xi: G \rightarrow \mathrm{GL}\left(\mathbf{V}^{*}\right)$ by

$$
\Xi(x) \nu:=\bar{m}\left(x^{-1}\right)^{t}\left(\operatorname{ev}_{1}\right)(\nu)(x)=\operatorname{ev}_{1}\left(\pi^{\vee}\left(x^{-1}\right)^{t} \operatorname{ev}_{1}(\nu)\right) .
$$

Theorem 1.3. Let the notation be as above. Then

(1) $\Xi(k x h)=\delta^{\vee}\left(h^{-1}\right) \Xi(x) \delta^{\vee}\left(k^{-1}\right)$.

(2) $\mathscr{K}_{x}(y)=\sum_{j=1}^{n} \bar{m}\left(y^{-1}, 1\right)^{-1} \Xi\left(x^{-1} y\right) \nu_{i} \otimes m\left(x^{-1}, 1\right)^{-1} v_{i}$.

(3) If $m \equiv 1$ and there is a measure $\mu$ on $G / K$ such that $(f \mid g)_{E}=$ $\int_{G / K}(f(x) \mid g(x))_{V} d \mu(x K)$, then $f(x)=\int_{G / K}{ }^{t} \Xi\left(x^{-1} y\right) f(y) d \mu(K)$ for all $f \in$ $\mathbf{E}$ and $x \in G$.

(4) If $G$ is a semisimple Lie group, $K$ a maximal compact subgroup, and $\mathbf{E}$ unitary and irreducible, then $\mathscr{K}_{x}$ is an analytic function for all $x \in G$.

Proof. (1) follows from the intertwining properties of ${ }^{t} \mathrm{ev}_{1}$. By Theorem 1.2, $\mathscr{K}_{x}(y)=\sum\left[\pi^{\vee}(x) \phi_{i}\right](y) \otimes m\left(x^{-1}\right)^{-1} v_{i}$. As $\pi$ is unitary,

$$
\left[\pi^{\vee}(x) \phi_{i}\right](y)=\bar{m}\left(y^{-1}\right)^{-1}\left[\pi^{\vee}\left(y^{-1} x\right) \phi_{i}\right](1)=\bar{m}\left(y^{-1}\right)^{-1} \Xi\left(x^{-1} y\right)\left(\nu_{i}\right) .
$$

For (3) we notice first that for $f, g \in \mathbf{E}, x \in G$, and $k \in K,(f(x k) \mid g(x k))_{V}$ $=\left(\rho\left(k^{-1}\right) f(x) \mid \rho\left(k^{-1}\right) g(x)\right)_{V}=(f(x) \mid g(x))_{V}$. Hence $x \mapsto(f(x) \mid g(x))_{V}$ is 
actually a function on $G / K$ and thus the integral makes sense. By our identification of $\mathbf{E}^{*}$ with $\mathbf{E}$ (resp. $\mathbf{V}^{*}$ with $\mathbf{V}$ ) we get $\left\langle f, j_{E} g\right\rangle=(f \mid g)_{E}=$ $\int_{G / K}(f(x) \mid g(x))_{V} d \mu(x K)=\int_{G / K}\left\langle f(x), j_{V}(g(x))\right\rangle d \mu(x K)$. Thus

$$
\begin{aligned}
f(x) & =\left\langle f, \mathscr{K}_{x}\right\rangle_{E}=\sum\left\langle f, \pi^{\vee}(x) \phi_{i}\right\rangle v_{i} \\
& =\sum \int_{G / K}\left\langle f(y), \Xi\left(x^{-1} y\right) \nu_{i}\right\rangle v_{i} d \mu(y K) \\
& =\sum \int_{G / K}\left\langle{ }^{t} \Xi\left(x^{-1} y\right) f(y), \nu_{i}\right\rangle v_{i} d \mu(y K) \\
& =\int_{G / K}{ }^{t} \Xi\left(x^{-1} y\right) f(y) d \mu(y K) .
\end{aligned}
$$

By Theorem 1.1 the functions $\phi_{i}$ are $K$-finite and-as $\mathbf{E}$ is irreducible$Z(\mathfrak{g})$-finite $(Z(\mathfrak{g})$ the center of $U(\mathfrak{g}))$. By well-known arguments (e.g. [21]) $\phi_{i}$ is analytic. By Theorem 1.2, $\mathscr{K}_{x}=\sum_{j=1}^{n} \pi^{\vee}(x) \phi_{i} \otimes m\left(x^{-1}, 1\right)^{-1} v_{i}$ and (4) follows.

Let now $\lambda$ be a representation of $G$ in a Hilbert space $\mathbf{F}$ and $U \in$ $\operatorname{Hom}_{G}(\mathbf{F}, \mathbf{E}), U \neq 0$. Then

$$
{ }^{t} U \otimes 1 \in \operatorname{Hom}_{G}\left(\mathbf{E}^{*} \otimes \mathbf{V}, \mathbf{F}^{*} \otimes \mathbf{V}\right) \text { and }\left\langle f,{ }^{t} U \otimes 1(T)\right\rangle_{F}=\langle U(f), T\rangle_{E}
$$

for all $f \in \mathbf{F}$ and $T \in \mathbf{E}^{*} \otimes \mathbf{V}$.

Lemma 1.4. Define $\psi_{x}:=\left({ }^{t} U \otimes 1\right)\left(\mathscr{K}_{x}\right) \in \mathbf{F}^{*} \otimes \mathbf{V}$ and $\psi:=\psi_{1}$. Then

(1) $\psi_{x}=\lambda^{\vee}(x) \otimes m\left(x^{-1}\right)^{-1} \psi$ and

$$
U f(x)=\left\langle f, \psi_{x}\right\rangle_{E}=m\left(x^{-1}\right)^{-1}\left\langle\lambda\left(x^{-1}\right) f, \psi\right\rangle_{E}, \quad f \in \mathbf{F} .
$$

(2) $\psi \in \mathbf{F}^{*}\left(\delta^{\vee}\right) \otimes \mathbf{V}$ and $\left(\lambda^{\vee}(k) \otimes 1\right) \psi=\left(1 \otimes \delta\left(k^{-1}\right)\right) \psi$. In particular, if $G$ is a semisimple Lie group, $K$ a maximal compact subgroup, and $\mathbf{F}^{*}$ is $Z(\mathfrak{g})$-finite, then $\psi \in \mathbf{F}_{\omega}^{*} \otimes \mathbf{V}$.

Proof. $U f(g)=\left\langle U f, \mathscr{K}_{g}\right\rangle_{E}=\left\langle f,\left({ }^{t} U \otimes 1\right) \mathscr{K}_{g}\right\rangle_{F}=\left\langle f, \psi_{g}\right\rangle_{F}, f \in \mathbf{F}$. The rest follows by the same arguments as in the proof of Theorem 1.3 and from the intertwining properties of ${ }^{t} U \otimes 1$, Theorem 1.2.

\section{THE DISCRETE SERIES OF $G$}

From now on $G$ will stand for a noncompact connected semisimple Lie group with Lie algebra $\mathfrak{g}_{0}$. In this paragraph we recall Hotta's construction of the discrete series ([9] and [23]) and prove that the evaluation map $\mathrm{ev}_{1}$ is continuous (see also [23] and [6]).

Let $\mathfrak{g}$ denote the complexification of $\mathfrak{g}_{0}$. If $\mathfrak{h}_{0}$ is a subspace of $\mathfrak{g}_{0}$ we identify the complexification $\mathfrak{h}$ of $\mathfrak{h}_{0}$ with the subspace of $\mathfrak{g}$ generated by $\mathfrak{h}_{0}$. If $t$ is an abelian Lie algebra, $V$ a semisimple $t$ module, and $\alpha \in \mathfrak{t}^{*}$, then $V_{\alpha}:=\{v \in V \mid \forall h \in \mathfrak{t}: h \cdot v=\alpha(h) v\}, \Delta(V, \mathfrak{t}):=\left\{\alpha \in \mathfrak{t}^{*} \mid \alpha \neq 0, V_{\alpha x} \neq 0\right\}$, and $\rho(\Gamma)=\frac{1}{2} \sum_{\alpha \in \Gamma}\left(\operatorname{dim}\left(V_{\alpha}\right)\right) \alpha, \varnothing \neq \Gamma \subset \Delta(V, \mathfrak{t})$. Let $B$ be the Killing form of $\mathfrak{g}$. 
If $\mathfrak{t}$ is a subspace of $\mathfrak{g}$ such that $B$ is nondegenerate on $\mathfrak{t}, \lambda \in \mathfrak{t}^{*}$, we define $h_{\lambda} \in \mathfrak{t}$ by $\lambda(x)=B\left(x, h_{\lambda}\right), x \in \mathfrak{t}$. For $\lambda, \mu \in \mathfrak{t}^{*}$ let $(\lambda, \mu):=B\left(h_{\lambda}, h_{\mu}\right)$.

Let $\vartheta$ be a Cartan involution of $G$; then $\vartheta$ stands for the corresponding involution on $\mathfrak{g}_{0}, \mathfrak{g}, \mathfrak{g}_{0}^{*}$, and $\mathfrak{g}^{*}$ too. Then $\mathfrak{g}_{0}=\mathfrak{k}_{0} \oplus \mathfrak{p}_{0}$ where $\mathfrak{k}_{0}$ is the Lie algebra of $K:=G^{\vartheta}$ and $\mathfrak{p}_{0}$ is the $(-1)$-eigenspace of $\vartheta$. Then $\mathfrak{k}_{0}$ is the $(+1)$ eigenspace of $\vartheta$, and $Z$, the center of $G$, is contained in $K$. Let $R:=G / K$, $G^{0}:=G / Z, K^{0}:=K / Z$, and normalize measures such that compact groups have total measure one and

$$
\int_{K} f(k) d_{K} k=\int_{K^{0}} \int_{Z} f(k z) d_{K} \circ k d_{Z} z, \quad f \in \mathscr{C}_{c}(K),
$$

and

$$
\begin{aligned}
\int_{G} f(x) d_{G} x & =\int_{R} \int_{K} f(y k) d_{R} y d_{K} k \\
& =\int_{G^{0}} \int_{Z} f(x z) d_{G^{0}} x d_{z} z, \quad f \in \mathscr{C}_{c}(G) .
\end{aligned}
$$

Let $\mathfrak{t}$ be a Cartan subalgebra of $\mathfrak{k}$. We will always assume that $\mathfrak{t}$ is also a Cartan subalgebra of $\mathfrak{g}$, i.e. $\operatorname{rank}(G)=\operatorname{rank}(K)$. Let $T \subset K$ be the corresponding Cartan subgroup and $\lambda \in i t_{0}^{*}$ regular. As $\mathfrak{p}$ is ad(t)-stable, $\Delta_{\mathfrak{g}}:=\Delta(\mathfrak{g}, \mathfrak{t})$, $\Delta_{\mathfrak{k}}:=\Delta(\mathfrak{k}, \mathfrak{t})$, and $\Delta_{\mathfrak{p}}:=\Delta(\mathfrak{p}, \mathfrak{t})$ are well-defined. Let

$$
\Delta_{\mathfrak{g}}^{+}:=\left\{\alpha \in \Delta_{\mathfrak{g}} \mid(\lambda, \alpha)>0\right\}, \quad \Delta_{\mathfrak{k}}^{+}:=\Delta_{\mathfrak{k}} \cap \Delta_{\mathfrak{g}}^{+}, \quad \Delta_{\mathfrak{p}}^{+}:=\Delta_{\mathfrak{p}} \cap \Delta_{\mathfrak{g}}^{+} .
$$

We also define $\rho_{\mathfrak{g}}:=\rho\left(\Delta_{\mathfrak{g}}^{+}\right), \rho_{\mathfrak{k}}:=\rho\left(\Delta_{\mathfrak{k}}^{+}\right)$, and $\rho_{\mathfrak{p}}:=\rho\left(\Delta_{\mathfrak{p}}^{+}\right)$. Assume that there is an irreducible representation $\delta_{\lambda}$ of $K$ with highest weight $\mu_{B}(\lambda):=$ $\lambda+\rho_{\mathfrak{g}}-2 \rho_{\mathfrak{k}}=\lambda+\rho_{\mathfrak{p}}-\rho_{\mathfrak{k}}$. Denote the representation space $\delta_{\lambda}$ by $\mathbf{V}_{\lambda}$ or simply by $\mathbf{V}$. We consider the associated homogeneous $\mathscr{C}^{\infty}$ vector bundle $\mathbb{V}_{\lambda}:=G \times_{K} \mathbf{V} \rightarrow R$ over $R$. Let $\mathbf{H}_{2}(\lambda)$ be the subspace of $\mathbf{L}^{2}$-sections, where $\widehat{\Omega}$, the closure of the Casimir element $\Omega \in Z(\mathfrak{g})$, acts by $\|\lambda\|^{2}-\left\|\rho_{\mathfrak{g}}\right\|^{2}$, i.e. $\mathbf{H}_{2}(\lambda)$ is the space of $\mathscr{C}^{\infty}$-functions of $G$ into $\mathbf{V}$ such that

(1) $\forall k \in K, \forall a \in G: f(a k)=\delta_{\lambda}\left(k^{-1}\right) f(a)$,

(2) $\widehat{\Omega} f=\left(\|\lambda\|^{2}-\left\|\rho_{\mathfrak{g}}\right\|^{2}\right) f$,

(3) $\|f\| \in \mathbf{L}^{2}(R)$.

Then $\mathbf{H}_{2}(\lambda)$ is a Hilbert space with inner product given by

$$
(f \mid g)=\int_{R}(f(x) \mid g(x)) d_{R}(x K)=\int_{G^{0}}(f(x) \mid g(x)) d_{G^{0}}(x Z) .
$$

$G$ acts irreducibly and unitarily on $\mathbf{H}_{2}(\lambda)$ by left translation and the corresponding representation $\pi_{\lambda}$ is unitary equivalent to the (relative) discrete series representation of $G$ associated to the Blattner parameter $\mu_{B}(\lambda)$ (see [9] and [23]).

Let $\chi:=\operatorname{dim}(\mathbf{V}) \cdot \operatorname{trace} \circ \delta_{\lambda}: K \rightarrow \mathbb{C}$ and let $\xi: Z \rightarrow \mathbb{C}$ be the central character of $\pi_{\lambda}$, i.e. $\pi_{\lambda} \mid Z=\zeta$. id. Choose $f \in \mathbf{H}_{2}(\lambda)$ such that $f(1) \neq 0$. Then

$$
\xi(z) f(1)=f\left(z^{-1}\right)=\delta_{\lambda}(z) f(1),
$$


and $\delta_{\lambda} \mid Z=\xi \cdot$ id and $\chi(k z)=\xi(z) \chi(k)$ for $z \in Z, k \in K$. Hence

$$
\operatorname{pr}(f)(x):=\int_{K^{0}} \overline{\chi(k)} f\left(k^{-1} x\right) d_{K^{0}}(k Z)
$$

is well-defined and pr is the continuous, orthogonal $K$-intertwining projection onto $\mathbf{H}_{2}(\lambda)\left(\delta_{\lambda}\right)$. Now

$$
\begin{aligned}
\operatorname{pr}(f)(1) & =\int \overline{\chi(k)} f\left(k^{-1}\right) d(k Z) \\
& =\int \overline{\chi(k)} \delta_{\lambda}(k) d(k Z) f(1) \\
& =f(1) .
\end{aligned}
$$

Thus $\mathrm{ev}_{1}=\left(\mathrm{ev}_{1} \mid \mathbf{H}_{2}(\lambda)\left(\delta_{\lambda}\right)\right) \circ \mathrm{pr}$. As the multiplicity of $\delta_{\lambda}$ in $\mathbf{H}_{2}(\lambda)$ is one $-\delta_{\lambda}$ is the lowest $K$-type of $\pi_{\lambda}$-we also have that $\operatorname{ev}_{1} \mid \mathbf{H}_{2}(\lambda)\left(\delta_{\lambda}\right): \mathbf{H}_{2}(\lambda)\left(\delta_{\lambda}\right) \rightarrow \mathbf{V}$ is a $K$-equivariant, continuous isomorphism, and $\mathrm{ev}_{1} \mid \mathbf{H}_{2}(\lambda)\left(\delta_{\lambda}\right)^{\perp}=0$. We have now proved

Theorem 3.1. $\mathrm{ev}_{x}: \mathbf{H}_{2}(\lambda) \rightarrow \mathbf{V}$ continuous for all $x \in G$.

By this theorem our results in $\S 1$ can be applied to the discrete series representations. In particular, Theorem 1.3 can be used to write down the reproducing kernel. Notice that the dual of $\mathbf{H}_{2}(\lambda)$ is $\mathbf{H}_{2}\left(-w^{*} \lambda\right)$ where $w^{*}$ is the unique element of the Weyl group of $\Delta_{\mathfrak{k}}$ such that $w^{*} \Delta_{\mathfrak{k}}^{+}=-\Delta_{\mathfrak{k}}^{+}$.

\section{THE DISCRETE SERIES OF $X$}

Let $\tau$ be a nontrivial involution of $G$ (and $\mathfrak{g}_{0}, \mathfrak{g}, \mathfrak{g}_{0}^{*}, \mathfrak{g}^{*}$ ), commuting with $\vartheta$ and let $H$ be an open subgroup of $G^{\tau}$. Then $H$ is a closed subgroup of $G$. Let $\mathfrak{h}_{0}$ be the Lie algebra of $H$ and $\mathfrak{q}_{0}$ the $(-1)$-eigenspace of $\tau$. Then $\mathfrak{g}=\mathfrak{h} \oplus \mathfrak{q}=\mathfrak{h}_{k} \oplus \mathfrak{q}_{k} \oplus \mathfrak{h}_{p} \oplus \mathfrak{q}_{p}$, where the subscript $k$ (resp. $p$ ) means the intersection with $\mathfrak{k}$ (resp. p). Let $X$ be the affine symmetric space $G / H$. We also define $H^{0}$ to be the connected component of $\left(G^{0}\right)^{\tau}$, and $X^{0}:=G^{0} / H^{0}$.

From now on we assume that $\mathfrak{t}$ is $\tau$-stable and that $\mathfrak{a}:=\mathfrak{t} \cap \mathfrak{q}$ is maximal abelian in $\mathfrak{q}$. Then the rank of $X$ is the same as the rank of $K / K \cap H$ and, by a theorem of Flensted-Jensen [3], the relative discrete series of $X$ is nonzero. For the construction and parametrization of the discrete series of $X$ we refer the reader to $[3,5,12,19]$ and the references there. We will only remind the reader that those representations are parametrized by a subset $\Lambda \subset i \mathrm{a}_{0}^{*}$. We denote the corresponding representation space by $\mathbf{F}_{\lambda}, \lambda \in \Lambda$. Let $\mathfrak{l}$ be the centralizer of $\mathfrak{a}$ in $\mathfrak{g}$ and $\Delta:=\Delta(\mathfrak{g}, \mathfrak{a}), \Delta_{c}:=\Delta(\mathfrak{k}, \mathfrak{a}), \Delta(\mathfrak{l}):=\Delta(\mathfrak{l}, \mathfrak{t})$, and $\Delta_{c}(\mathfrak{l}):=\Delta\left(\mathfrak{l}_{k}, \mathfrak{t}\right)$. Choose a positive ordering in $i t_{0}^{*}$ such that $i \mathrm{a}_{0}^{*}$ comes first and let the superscript + denote the corresponding positive system of roots. Let $\rho:=\rho\left(\Delta^{+}\right)$and $\rho_{c}:=\rho\left(\Delta_{c}^{+}\right)$, and define for $\lambda \in \mathfrak{a}^{*}, \mu_{F}(\lambda):=\lambda+\rho-2 \rho_{c}$. Then $\mu_{F}(\lambda)=\mu_{B}\left(\lambda-\rho\left(\Delta^{+}(\mathfrak{l})\right)+2 \rho\left(\Delta^{+}\left(\mathfrak{l}_{k}\right)\right)\right)$.

Definition 3.1. The pair $\left(\mathfrak{g}_{0}, \tau\right)$ (or the space $X$ ) is said to satisfy Condition $\mathrm{D}$, if the centralizer of $\mathfrak{a}_{0}$ in $G^{0}$ is compact. 
The reader can find a list of the simple classical spaces satisfying Condition $\mathrm{D}$ in [17], from which this definition is taken. Notice, also, that $X$ satisfies Condition $\mathrm{D}$ if $X$ is of Hermitian type (see Definition 5.1).

If $X$ satisfies Condition $\mathrm{D}$, then $\mathfrak{l}=\mathfrak{l}_{k}$ and $\rho(\mathfrak{l})=\rho_{c}(\mathfrak{l})$. Thus $\mu_{F}(\lambda)=$ $\mu_{B}(\lambda+\rho(\mathfrak{l}))$ and $\lambda+\rho(\mathfrak{l})$ is dominant regular, if $(\lambda, \alpha)>|(\rho(\mathfrak{l}), \alpha)|$ for all $\alpha \in \Delta_{\mathfrak{g}}^{+}$. In that case the discrete series representation in $\mathbf{H}_{2}(\lambda+\rho(\mathfrak{l}))^{*}$ exists and is equivalent to the representation of $G$ in $\mathbf{F}_{\lambda}$ by [18] and [13]. Thus infinitely many of the representations $\mathbf{F}_{\lambda}$ can be realized in the discrete series of $G$.

We will now assume that $\lambda \in i t_{0}^{*}$ is such that there is a unitary intertwining operator $T: \mathbf{H}_{2}(\lambda) \rightarrow \mathbf{L}_{\xi}^{2}(X)$, where $\xi$ is determined by $\xi \cdot$ id $=\pi_{\lambda} \mid Z$ and $\mathbf{L}_{\xi}^{2}(X)$ is the space of measurable functions $f$ on $X$, such that $l_{z} f=\xi(z) f$, $z \in Z$, and $|f| \in \mathbf{L}^{2}\left(X^{0}\right)$. Let $\mathbf{E}_{\lambda}:=\operatorname{Im}(T), U:=T^{-1}: \mathbf{E}_{\lambda} \rightarrow \mathbf{H}_{2}(\lambda)$, and $\varepsilon_{\lambda}$ be the representation of $G$ on $\mathbf{E}_{\lambda}$. Notice that $\overline{\mathbf{E}}_{\lambda}=\left\{\bar{f} \mid f \in \mathbf{E}_{\lambda}\right\}$ is the dual of $\mathbf{E}_{\lambda}$. Let $\mathscr{A}\left(X, \delta_{\lambda}, \chi\right)$ be the space of functions $f: X \rightarrow \mathbf{V}$ such that

(1) $\forall k \in K, \forall x \in X: f(k x)=\delta_{\lambda}(k) f(x)$,

(2) $\forall z \in Z(\mathfrak{g}): z f=\chi(z) f$, where $\chi: Z(\mathfrak{g}) \rightarrow \mathbb{C}$ is the infinitesimal character of $\pi_{\lambda}^{\vee}$.

If $f \in \mathscr{A}\left(X, \delta_{\lambda}, \chi\right) \cap \mathbf{L}_{\bar{\xi}}(X)$ then $f$ is analytic and bounded [1, pp. 230, 244].

Theorem 3.2. Let the notation be as above. Let $\mathscr{K}$ be the reproducing kernel of $\pi_{\lambda}$ and define $\phi: X \rightarrow \mathbf{V}$ by $\phi:=\left({ }^{t} U \otimes 1\right) \mathscr{K}_{1}$. Then $\phi$ has the following properties, where $a, b \in G$ and $x \in X$ :

(1) $\phi \in \mathscr{A}\left(X, \delta_{\lambda}, \chi\right) \cap \overline{\mathbf{E}}_{\lambda} \otimes \mathbf{V} \subset \mathbf{L}_{\bar{\xi}}^{2}(X, \mathbf{V})$.

(2) $\forall f \in \mathbf{E}_{\lambda}: U f(a)=\left\langle f, \varepsilon_{\lambda}^{\vee} \otimes 1(a) \phi\right\rangle_{E_{\lambda}}=\int_{X^{0}} f(x) \phi\left(a^{-1} x\right) d_{X^{0}} x$,

(3) ${ }^{t} T \otimes 1\left(\varepsilon_{\lambda}^{\vee} \otimes 1(a) \phi\right)(b)=\mathscr{K}(a, b):=\mathscr{K}_{a}(b)$.

(4) Define for $\nu \in \mathbf{V}^{*}, \phi_{\nu}(x):=\langle\phi(x), \nu\rangle$. Then $\phi_{\nu} \in \overline{\mathbf{E}}_{\lambda}\left(\delta_{\lambda}^{\vee}\right)$ and $\mathbf{V}^{*} \ni$ $\nu \mapsto \phi_{\nu} \in \overline{\mathbf{E}}_{\lambda}\left(\delta_{\lambda}^{\vee}\right)$ is a K-isomorphism.

Proof. Everything except (4) follows from Lemma 1.4. By $(2), \phi_{\nu}\left(k^{-1} x\right)=$ $\left\langle\phi\left(k^{-1} x\right), \nu\right\rangle=\left\langle\delta_{\lambda}\left(k^{-1}\right) \phi(x), \nu\right\rangle=\left\langle\phi(x), \delta_{\lambda}^{\vee}(k) \nu\right\rangle=\phi_{\delta_{\lambda}^{\vee}(k) \nu}(x)$. As $\phi(x)$ is nonzero for some $x \in X$, we can find a $\nu$ such that $\phi_{\nu} \neq 0$. Thus $\nu \mapsto \phi_{\nu}$ is a nonzero $K$-map. As $\delta_{\lambda}^{\vee}$ is irreducible and its multiplicity in $\overline{\mathbf{E}}_{\lambda}$ is one, the theorem follows.

If $\lambda \in i \mathrm{a}_{0}^{*}$ and $\lambda+\rho(\mathfrak{l})$ is dominant and regular, then $\overline{\mathbf{F}}_{\lambda}$ is generated by the Flensted-Jensen function $\psi_{\lambda}$, which is $H \cap K$-invariant, of type $\delta_{\lambda+\rho(1)}^{\vee}$, and $\psi_{\lambda}(1)=1$. By Theorem 3.2(4) there is a $\nu \in \mathbf{V}^{*}$ such that $\phi_{\nu}=\psi_{\lambda}$. As $\psi_{\lambda}$ is $H \cap K$-invariant it follows that $\nu$ is $H \cap K$-invariant and that $\phi(1 H)$ is a nonzero $H \cap K$-invariant vector in $\mathbf{V}$. We collect this in

Theorem 3.3. If $\lambda \in i \mathrm{a}_{0}^{*}$ and $\lambda+\rho(\mathfrak{l})$ is dominant and regular, then there is a $\nu \in \mathbf{V}^{* H \cap K}$ such that $\psi_{\lambda}=\langle\Phi(\cdot), \nu\rangle$. Furthermore $\Phi(1 H) \in \mathbf{V}^{H \cap K} \backslash\{0\}$. 


\section{THE INTERTWINING OPERATOR}

In this section we will show that on the level of $K$-finite functions, the intertwining operator $T: \mathbf{H}_{2}(\lambda) \rightarrow \mathbf{E}_{\lambda}$ is determined by the function $y \mapsto \phi\left(y^{-1} x\right)$ if $\pi_{\lambda}$ is integrable.

Definition 4.1. Let $\pi$ be a unitary irreducible representation of $G$. Then $\pi$ is said to be integrable if $x \mapsto|(\pi(x) u \mid v)|$ is integrable on $G^{0}$ for $u, v K$-finite.

According to [7] and [20], $\pi_{\lambda}$ is integrable if

$$
|(\lambda, \beta)|>\frac{1}{2} \sum_{\alpha \in \Delta_{\mathfrak{g}}^{+}}|(\beta, \alpha)|, \quad \text { for all } \beta \in \Delta_{\mathfrak{p}}^{+} .
$$

In that case $\|f(\cdot)\| \in \mathbf{L}^{1}(R)$ for $f \in \mathbf{H}_{2}(\lambda) \quad K$-finite [23, p. 268].

Let the notation be as in $\S 3$. In particular, $T$ is a unitary intertwining operator from $\mathbf{H}_{2}(\lambda)$ into $\mathbf{E}_{\lambda} \subset \mathbf{L}_{\xi}^{2}(X)$ with inverse $U$, and $\phi \in \mathscr{A}\left(X, \delta_{\lambda}, \chi\right) \cap$ $\overline{\mathbf{E}}_{\lambda} \otimes \mathbf{V}$ is such that $U(f)(a)=\int f(x) \phi\left(a^{-1} x\right) d_{X^{0}} x$.

Theorem 4.2. Assume that $\pi_{\lambda}$ is integrable and $f \in \mathbf{H}_{2}(\lambda) \quad K$-finite. Then

$$
\begin{aligned}
T(f)(x) & =\int_{G^{0}}\left(f(a) \mid \phi\left(a^{-1} x\right)\right)_{V} d_{G^{0}} a \\
& =\int_{R}\left(f(a) \mid \phi\left(a^{-1} x\right)\right)_{V} d_{R}(a K) .
\end{aligned}
$$

Proof. That the two integrals are equal follows from Theorem 3.2(1). As $\phi$ is bounded

$$
\int_{R}\left|\left(f(a) \mid \phi\left(a^{-1} x\right)\right)_{V}\right| d_{R}(a K) \leq \sup \|\phi(x)\| \int_{R}\|f(a)\|_{V} d_{R}(a K)<\infty .
$$

Hence the integral exists for all $f K$-finite. Denote it by $T_{1} f(x)$. Let $g \in \overline{\mathbf{E}}_{\lambda}$; then

$$
\begin{aligned}
& \iint\left|\left(f(a) \mid \phi\left(a^{-1} x\right)\right)_{V} g(x)\right| d_{R}(a K) d_{X} x \\
& \quad \leq \iint\|f(a)\|\left\|\bar{g}(x) \phi\left(a^{-1} x\right)\right\| d_{X} x d_{R}(a K) \\
& \quad \leq\|f\|_{\mathbf{L}^{1}}\|g\|\|\phi\|<\infty .
\end{aligned}
$$

Thus $T_{1} f$ defines a continuous linear form on $\overline{\mathbf{E}}_{\lambda}$ and the function $(a, x) \mapsto$ $g(x)\left(f(a) \mid \phi\left(a^{-1} x\right)\right)_{V}$ is integrable on $R \times X$. By Riesz' Representation Theorem there exists a function $\gamma \in \mathbf{E}_{\lambda}$ such that

$$
\int g(x) \gamma(x) d_{X^{0}}(x)=\int g(x) T_{1} f(x) d_{X^{0}}(x)
$$

for all $g \in \overline{\mathbf{E}}_{\lambda}$. By Fubini's theorem we get

$$
\int g(x) \gamma(x) d_{X^{0}}(x)=(f \mid U \bar{g})_{H_{2}(\lambda)}=(T f \mid \bar{g})_{E_{i}} .
$$


Thus $\gamma$ is $K$-finite and analytic. Let $g \in \mathscr{C}_{c}(X)$ and define

$$
g^{0}(x):=\int_{Z} \overline{\xi(z)} g(z x) d_{Z} z
$$

Then $g^{0} \in L_{\bar{\xi}}^{2}(X)$. Let $p: \mathbf{L}_{\bar{\xi}}^{2}(X) \rightarrow \overline{\mathbf{E}}_{\lambda}$ be the orthogonal projection. Then, by using Fubini's theorem again,

$$
\int g(x)\left(\gamma(x)-T_{1} f(x)\right) d x=\left(f \mid U\left(\overline{p g^{0}}\right)-U\left(\overline{p g^{0}}\right)\right)_{H_{2}(\lambda)}=0 .
$$

Thus $\gamma(x)=T_{1} f(x)$ for almost all $x \in X$. As both functions are continuous the claim follows.

\section{THE HOLOMORPHIC DISCRETE SERIES OF $X^{\#}$}

In the general case there is no explicit formula for the function $\phi$ on $X$. The best one can say is that there is a vector-valued distribution $F$ on the boundary of the noncompact Riemannian form $X^{r}=G^{d} / K^{d}$ such that $\phi$ is given by the Poisson transform of $F$ followed by the Flensted-Jensen isomorphism. Here $G^{d}$ is a connected Lie group with Lie algebra $\mathfrak{g}_{0}^{d}=\mathfrak{h}_{0 k} \oplus i \mathfrak{h}_{0 p} \oplus i \mathfrak{q}_{0 k} \oplus \mathfrak{q}_{0 p}$ as usual (see [5] for notation). But if $X$ is of Hermitian type and $\pi_{\lambda}$ is a holomorphic discrete series representation, we can use this to give a formula analogous to our results in [14, Theorem 6.2] for the scalar holomorphic discrete series. In this section we give the general construction of the holomorphic discrete series of the universal covering $X^{\#}$ of $X$ and relate those with the discrete series of Flensted-Jensen.

We assume from now on that $G$ is contained in a simply-connected, complex Lie group $G_{c}$ with the Lie algebra $\mathfrak{g}$. Let $G^{\#}$ denote the simply-connected covering of $G$ and let $K^{\#}:=G^{\# \vartheta}, H^{\#}:=G^{\# \tau}, K_{c}:=G_{c}^{\vartheta}$, and $H_{c}:=G_{c}^{\tau}$. As $G^{\#}$ and $G_{c}$ are simply-connected, all of those groups are connected, (this follows from [8, Theorem 8.2]). Let $K_{c}^{\#}$ be the simply-connected covering of $K_{c}$. Then we may identify $K^{\#}$ with the analytic subgroup of $K_{c}^{\#}$ corresponding to $\mathfrak{k}_{0}$. Denote the canonical covering maps $G^{\#} \rightarrow G, K^{\#} \rightarrow K, K_{c}^{\#} \rightarrow K_{c}$ in all cases by $\kappa$. Then $\kappa$ also defines a covering map $X^{\#} \rightarrow X, X^{\#}:=G^{\#} / H^{\#}$, and $X^{\#}$ is simply-connected. With our usual notation, $G$ a noncompact semisimple Lie group with Lie algebra $\mathfrak{g}_{0}, \tau$ a nontrivial involution of $G$ (and $\mathfrak{g}_{0}$ ), and $X=G / H$ with $H$ an open subgroup of $G^{\tau}$, we now define

5.1. Definition. The pair $\left(\mathfrak{g}_{0}, \tau\right)$-or the space $X$-is said to be of Hermitian type, if the following holds:

(1) There is no noncompact ideal in $\mathfrak{g}_{0}$ contained in $\mathfrak{h}_{0}$.

(2) Let $\mathfrak{c}$ be the centralizer of $\mathfrak{q}_{k}$ in $\mathfrak{q}_{k}$; then $\mathfrak{z}_{q}(\mathfrak{c})=\mathfrak{q}_{k}$.

Notice that this is weaker than the definition in [14], but the results of [14] still hold. In particular this is true for the structure theory in $\S 1$ and $\S 2$ of [14]. If $X$ is of Hermitian type, then $\mathfrak{c}$ is nonzero and contained in the center of $\mathfrak{k}$. 
Furthermore $\mathfrak{z}_{\mathfrak{g}}(\mathfrak{c})=\mathfrak{k}$ and $R$ is a bounded symmetric domain. In particular we can choose $\mathfrak{a}$ and $\mathfrak{t}$ such that $\mathfrak{c} \subset \mathfrak{a} \subset \mathfrak{t} \subset \mathfrak{k}$. Choose a basis $h_{1}, \ldots, h_{r}$ of $i \mathrm{t}_{0}$ such that the first $p$ elements are a basis of $i \mathfrak{c}_{0}$, the first $q$ elements are a basis of $i \mathfrak{a}_{0}$, and the rest are in $i t_{0} \cap \mathfrak{l}_{0}$. Our ordering is then the lexicographic ordering relative to this basis. If $\alpha \in \Delta$, then $\mathfrak{g}_{\alpha}$ is in $\mathfrak{k}$ or $\mathfrak{p}$. Let $\Delta_{n}^{+}$be the set of positive roots such that $\mathfrak{g}_{\alpha} \subset \mathfrak{p}$, and let $\mathfrak{p}^{ \pm}:=\bigoplus \mathfrak{g}_{ \pm \alpha}, \alpha \in \Delta_{n}^{+}$. Then $\mathfrak{p}^{+}$ and $\mathfrak{p}^{-}$are abelian algebras, $\mathfrak{g}=\mathfrak{p}^{+} \oplus \mathfrak{k} \oplus \mathfrak{p}^{-}$, and $\left[\mathfrak{k}, \mathfrak{p}^{ \pm}\right] \subset \mathfrak{p}^{ \pm}$.

Let $P^{ \pm}:=\exp \left(\mathfrak{p}^{ \pm}\right)$. Then $P^{+} \cap K_{c} P^{-}=\{1\}$ and $G \subset P^{+} K_{c} P^{-} \cap H_{c} K_{c} P^{+}=$: $O$. For $x \in O$ we define $p^{+}(x) \in P^{+}, k_{c}(x) \in K_{c}, q(x) \in P^{-}$, and $k_{H}(x) \in$ $H_{c} \cap K_{c} \backslash K_{c}$ by

$$
x=p^{+}(x) k_{c}(x) q^{-}(x) \in H_{c} k_{H}(x) P^{+} .
$$

$R$ is realized as a bounded symmetric domain $D$ in $\mathfrak{p}^{+}$by $x \mapsto z(x):=$ $\log \left(p^{+}(x)\right)$. Choose a maximal set of strongly orthogonal roots $\alpha_{1}, \ldots, \alpha_{s} \in \Delta_{n}^{+}$ as in $[14, \S 2]$. Choose $t_{i} \in \mathfrak{a}, x_{i} \in \mathfrak{p}_{\alpha_{i}}$, and $y_{i} \in \mathfrak{q}_{0 p}$ such that $\tau\left(\bar{x}_{i}\right)=$ $-x_{i},-$ the conjugation of $\mathfrak{g}$ relative to $\mathfrak{g}_{0},\left[x_{i}, \bar{x}_{i}\right]=t_{i}, \alpha_{i}\left(t_{i}\right)=2$, and $y_{i}=x_{i}-\tau\left(x_{i}\right)$. Then $\mathfrak{b}_{0}:=\sum_{i=1}^{s} \mathbb{R} y_{i}$ is maximal abelian in $\mathfrak{q}_{0 p}$ [14, Lemma 2.3]. Let $B$ be the corresponding analytic subgroup of $G\left(G^{*}\right.$ and $\left.G_{c}\right)$. If $b=\prod \exp \left(s_{i} y_{i}\right)$ then by [14, Theorem 2.4]

$$
k_{H}\left(b^{-1}\right)=\prod_{i=1}^{s} \exp \left(\frac{1}{2} \log \left(\cosh \left(2 s_{i}\right)\right) t_{i}\right) .
$$

Define $\gamma: X \rightarrow K_{c} / K_{c} \cap H_{c}$ by $\gamma(x H):=k_{H}\left(x^{-1}\right)^{-1}$. As $X^{\#}$ is simplyconnected, $\gamma$ lifts to $\gamma^{\#}: X^{\#} \rightarrow K_{c}^{\#} / K_{c}^{\# \tau}$ such that the diagram

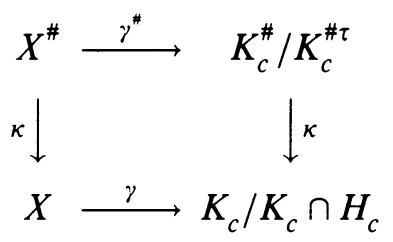

commutes and $\gamma^{\#}\left(1 H^{\#}\right)=1 K_{c}^{\# \tau}$. For $k \in K^{\#}, x \mapsto k^{-1} \gamma^{\#}(k x)$ defines another lifting (as $K_{c}$ normalizes $P^{+}$). Thus we find $e_{k}$ such that $k^{-1} \gamma^{\#}(k x)=e_{k} \gamma^{\#}(x)$ for all $x$. As $k^{-1} \gamma^{\#}(k)=e_{k} K_{c}^{\# \tau}$, the function $K^{\#} \ni k \mapsto e_{k} K_{c}^{\# \tau} \in \kappa^{-1}\left(1 K_{c}\right)$ is continuous, $\kappa^{-1}\left(1 K_{c}\right)$ is discrete, and $e_{1} K_{c}^{\# \tau}=1 K_{c}^{\# \tau}$. Thus $\gamma^{\#}(k x)=k \gamma^{\#}(x)$.

Let $\delta$ be a holomorphic representation of $K_{c}^{\#}$ in the finite dimensional Hilbert space $\mathbf{V}$. Assume that $\delta \mid K^{\#}$ is unitary and that $\delta$ contains a nonzero $K_{c}^{\# \tau}$-fixed vector. Let $\delta^{\vee}$ be the contragredient representation and $\nu^{0} \in \mathbf{V}^{*}$ $K_{c}^{\# \tau}$-invariant, $\nu^{0} \neq 0$. Define $\Phi_{\delta}=\Phi: X^{\#} \rightarrow \mathbf{V}^{*}$ by

$$
\Phi(x):=\delta^{\vee}\left(\gamma^{\#}(x)\right) \nu^{0} \text {. }
$$

Then $\Phi$ is analytic and $\Phi(k x)=\delta^{\vee}(k) \Phi(x), x \in X^{\#}, k \in K^{\#}$. If $Z^{\#}$ is the center of $G^{\#}$, and if $\xi \in \widehat{Z}^{\#}$ is defined by $\delta \mid Z^{\#}=\xi$ id, then $l_{z} \Phi=\xi(z) \Phi$, 
$z \in Z^{\#}$. For $v \in \mathbf{V}$ we let $\Phi_{v}:=\langle v, \Phi(\cdot)\rangle$, i.e. $\Phi_{v}(x)=\left\langle\delta\left(\gamma^{\#}(x)^{-1}\right) v, \nu^{0}\right\rangle=$ $\left\langle\delta\left(k_{c}^{\#}\left(x^{-1}\right)\right) v, \nu^{0}\right\rangle$, where $k_{c}^{\#}$ is defined in the obvious way. Then $l_{k} \Phi_{v}=$ $\Phi_{\delta(k) v}$, i.e. $\Phi_{v}$ is of type $\delta$.

Let $\mathfrak{n}^{ \pm}:=\sum_{\alpha \in \Delta^{+}} \mathfrak{g}_{ \pm \alpha}$ and $\mathfrak{n}_{c}^{ \pm}:=\mathfrak{n}^{ \pm} \cap \mathfrak{k} ;$ then $\mathfrak{n}^{ \pm}=\mathfrak{n}_{c}^{ \pm} \oplus \mathfrak{p}^{ \pm}$and

$$
\begin{aligned}
U(\mathfrak{g}) & =U(\mathfrak{t}) \oplus\left(\mathfrak{n}^{-} U(\mathfrak{g})+U(\mathfrak{g}) \mathfrak{n}^{+}\right) \\
& =U(\mathfrak{k}) \oplus\left(\mathfrak{p}^{-} U(\mathfrak{g})+U(\mathfrak{g}) \mathfrak{p}^{+}\right), \\
U(\mathfrak{k}) & =U(\mathfrak{t}) \oplus\left(\mathfrak{n}_{c}^{-} U(\mathfrak{k})+U(\mathfrak{k}) \mathfrak{n}_{c}^{+}\right) .
\end{aligned}
$$

Let $q^{\mathfrak{g}}, q_{\mathfrak{k}}$, and $q^{\mathfrak{k}}$ be the corresponding projection onto the first factor. If we need to emphasize the dependence on the choice of positive roots we will write $q^{\mathfrak{g}}\left(\Delta^{+}(\mathfrak{g}, \mathfrak{t})\right)$, etc. Then $q^{\mathfrak{g}}=q^{\mathfrak{k}} \circ q_{\mathfrak{k}}$. For $\lambda \in \mathfrak{t}^{*}$ we define $l_{\lambda}: S(\mathfrak{t}) \rightarrow S(\mathfrak{t})$ by $l_{\lambda}(h)=h-\lambda(h)$. For $\mathfrak{l}=\mathfrak{g}$ or $\mathfrak{k}$ we define $\mu^{\mathfrak{l}}:=l_{\rho_{\mathfrak{l}}} \circ q^{\mathfrak{l}}$. Then $\mu^{\mathfrak{l}}: Z(\mathfrak{l}) \rightarrow$ $S(\mathfrak{t})^{W(\mathfrak{l}, \mathfrak{t})}$ is an isomorphism, $q_{\mathfrak{k}}(Z(\mathfrak{g})) \subset Z(\mathfrak{k})$, and $\mu^{\mathfrak{g}}=l_{\rho_{n}} \circ \mu^{\mathfrak{k}} \circ p_{\mathfrak{k}}$. For $\lambda \in \mathfrak{t}$ define $\chi_{\lambda}^{\mathfrak{l}}: Z(\mathfrak{l}) \rightarrow \mathbb{C}$ by $\chi_{\lambda}^{\mathfrak{l}}(z):=\mu^{\mathfrak{l}}(z)(\lambda)$. Then every character of $Z(\mathfrak{l})$ is equal to some $\chi_{\lambda}$ and $\chi_{\lambda}=\chi_{\mu}$ if and only if there is a $w \in W(\mathfrak{l}, \mathfrak{t})$ such that $\lambda=w \cdot \mu$ and $\chi_{\lambda}$ is independent of the system of positive roots used to construct it.

Theorem 5.2. Let the notation be as above. Let $\mu$ be the highest weight of $\delta$ and assume $\langle\mu+\rho, \alpha\rangle<0$ for all $\alpha \in \Delta_{n}^{+}$. Then the following holds:

(1) $\Phi \in \mathscr{A}\left(X^{\#}, \delta^{\vee}, \chi_{\lambda+\rho_{\mathfrak{g}}}\right) \cap \mathbf{L}_{\xi}^{2}\left(X^{\#}\right) \otimes \mathbf{V}^{*}$ and $\mathfrak{p}^{+} \Phi=0$.

(2) Let $b=\exp \left(\sum s_{j} y_{j}\right) \in B$. Then

$$
\Phi_{v}(b)=\left\langle\delta\left(\prod_{i=1}^{s} \exp \left(\frac{1}{2} \log \left(\cosh \left(2 s_{i}\right)\right) t_{i}\right)\right) v, \nu^{0}\right\rangle \text {. }
$$

If $v$ is of weight $\nu$, then

$$
\Phi_{v}(b)=\left\langle v, \nu^{0}\right\rangle \prod \cosh \left(2 s_{i}\right)^{1 / 2 \nu\left(t_{i}\right)} .
$$

(3) $\Phi_{v} \in \mathbf{L}_{\xi}^{2}\left(X^{\#}\right)$.

(4) Let $\mathbf{H}_{\delta}(\boldsymbol{\delta}):=\left\{\Phi_{v} \mid v \in \mathbf{V}\right\}$ and $\mathbf{H}_{\delta}$ be the closure of $U(\mathfrak{g}) \mathbf{H}_{\delta}(\boldsymbol{\delta})$ in $\mathbf{L}_{\xi}^{2}\left(X^{\#}\right)$. Then $\mathbf{H}_{\delta}$ is irreducible, with infinitesimal character $\chi_{\lambda+\rho_{\mathrm{g}}}, \mathbf{V} \rightarrow$ $\mathbf{H}_{\delta}(\delta), v \mapsto \Phi_{v}$, is a $K^{\#}$-isomorphism, and $\mathbf{H}_{\delta}(\delta)$ is the lowest $K^{\#}$-type of $\mathbf{H}_{\delta}$.

(5) $\mathfrak{p}^{+} \mathbf{H}_{\delta}(\delta)=0$.

(6) If $\nu$ is a highest weight of a $K^{\#}$-type occurring in $\mathbf{H}_{\delta}$, then there exist $n_{\alpha} \in \mathbb{Z}^{+}, \alpha \in \Delta_{n}^{+}$, such that $\nu=\mu-\sum n_{\alpha} \alpha$.

Definition 5.3. The representations $\varepsilon_{\delta}$ on $\mathbf{H}_{\delta}$ are called the holomorphic discrete series of $X^{\#}$-or $X$ if $\mathbf{H}_{\delta} \subset \mathbf{L}^{2}(X)$-with lowest $K^{\#}$-type $\delta$. 
Proof of the theorem. Assume first that (1) holds. Then (3) and (5) are obvious. Furthermore (2) follows from (5.1) and the definition of $\Phi$. By (5) the HarishChandra module of $\mathbf{H}_{\delta}$ is isomorphic to $U\left(\mathfrak{p}^{-}\right) \otimes \mathbf{H}_{\delta}(\delta)$ as a representation of $K^{\#}$, and (6) follows. By (6) the multiplicity of $\delta$ in $\mathbf{H}_{\delta}$ is one and-as every nonzero vector in $\mathbf{H}_{\delta}(\delta)$ is cyclic and $\mathbf{H}_{\delta}$ is unitary- $\mathbf{H}_{\delta}$ is irreducible.

Now we prove (1). Let $x \in X^{\#}$. Then we can find an open neighbourhood $\mathscr{U}$ of $x$ and an open subset $\mathscr{O} \subset X$ such that $\kappa: \mathscr{U} \rightarrow \mathscr{O}$ is an analytic diffeomorphism. Define $\Psi: \mathscr{O} \rightarrow \mathbf{V}^{*}$ by $\Psi(\kappa(y))=\Phi(y), y \in \mathscr{U}$. Then, obviously, $X \Psi(\kappa(x))=X \Phi(x)$ for all $X \in \mathfrak{g}$. But by construction there exists an open subset $\mathscr{W} \subset P^{+} K_{c} / H_{c} \subset G_{c} / H_{c}$ such that $\Psi$ extends to a holomorphic function on $\mathscr{W}$ and $\Psi\left(p k H_{c}\right)=\Psi\left(k H_{c}\right), p \in P^{+}$and $k \in K_{c}$. As $\Psi$ is holomorphic, $X \Psi(y)=\left.\frac{d}{d t} \Psi(\exp (-t X) y)\right|_{t=0}$ for all $X \in \mathfrak{g}$. Hence $\mathfrak{p}^{+} \Psi(\kappa(x))=\mathfrak{p}^{+} \Phi(x)=0$. As $x$ was arbitrary,

$$
\mathfrak{p}^{+} \Phi=0 \text {. }
$$

Define now an antihomomorphism $u \mapsto u^{\vee}$ of $U(\mathfrak{g})$ by $X^{\vee}=-X, X \in \mathfrak{g}$. Let $v \in \mathbf{V}$ be a highest weight vector. Then for $z \in Z(\mathfrak{g})$,

$$
\begin{aligned}
\langle v, z \Phi(x)\rangle & =\left\langle v, q_{\mathfrak{k}}(z) \Phi(x)\right\rangle \\
& =\left\langle v, \delta^{\vee}\left(q_{\mathfrak{k}}(z)^{\vee}\right) \Phi(x)\right\rangle \\
& =\left\langle\delta\left(q_{\mathfrak{k}}(z)\right) v, \Phi(x)\right\rangle \\
& =\left\langle\delta\left(q^{\mathfrak{k}}\left(q_{\mathfrak{k}}(z)\right)\right) v, \Phi(x)\right\rangle \\
& =\mu\left(q^{\mathfrak{g}}(z)\right)\langle v, \Phi(x)\rangle \\
& =\chi_{\mu+\rho_{\mathfrak{g}}}(z)\langle v, \Phi(x)\rangle .
\end{aligned}
$$

As $v$ is cyclic and $q_{\mathfrak{k}}(z) \in Z(\mathfrak{k})$, it follows that $z \Phi=\chi_{\mu+\rho_{\mathfrak{g}}}(z) \Phi$.

That $\Phi_{v} \in \mathbf{L}_{\xi}^{2}(X)$ follows from the next theorem, which relates our construction with that of Flensted-Jensen, but we will give an independent proof here. For that we define the Cayley-transform $c:=\operatorname{Ad}\left(\exp \left(-\frac{\pi}{4} \sum\left(x_{j}-\bar{x}_{j}\right)\right)\right)$ and $\mathfrak{a}_{0}^{-}:=\sum \mathbb{R} i t_{j}$. Then $c\left(t_{j}\right)=y_{j}, c\left(i \mathfrak{a}_{0}^{-}\right)=\mathfrak{b}_{0}$, and ${ }^{t}\left(c^{-1}\right)\left(\Delta \mid i \mathfrak{a}_{0}^{-}\right) \backslash 0=$ $\Delta\left(\mathfrak{g}_{0}, \mathfrak{b}_{0}\right)$. Let $\Delta^{+}\left(\mathfrak{g}_{0}, \mathfrak{b}_{0}\right):={ }^{t}\left(c^{-1}\right)\left(\Delta^{+} \mid i \mathfrak{a}_{0}^{-}\right) \backslash 0$ and $\rho^{0}:=\rho\left(\Delta^{+}\left(\mathfrak{g}_{0}, \mathfrak{b}_{0}\right)\right)$. Then $\rho^{0}=\rho \circ c^{-1}$. In particular $\rho^{0}\left(y_{j}\right)=\rho\left(t_{j}\right)$. We only have to show that $\Phi_{v}, v$ of weight $\lambda$, is in $\mathbf{L}_{\xi}^{2}(X)$. By Lemma 5.5, which follows below, $\lambda\left(t_{j}\right)<-\rho\left(t_{j}\right)-\varepsilon \quad(\varepsilon>0)$ for all $j$. Let $b$ be as in (2) with $s_{j} \geq 0$ $(j=1,2, \ldots, s)$. Then

$$
\begin{aligned}
\left|\Phi_{v}(b)\right| & =\left\langle v, \nu_{0}\right\rangle \prod \cosh \left(2 s_{j}\right)^{1 / 2 \lambda\left(t_{j}\right)} \\
& \leq\left\langle v, \nu_{0}\right\rangle \prod \cosh \left(2 s_{j}\right)^{-1 / 2 \rho\left(t_{j}\right)-\varepsilon} \\
& \leq\left\langle v, \nu_{0}\right\rangle C \exp \left(-\rho\left(\sum s_{j} t_{j}\right)\right) \exp \left(-\varepsilon \sum s_{j}\right) \\
& =\left\langle v, \nu_{0}\right\rangle C \exp \left(-\rho^{0}\left(\sum s_{j} y_{j}\right)\right) \exp \left(-\varepsilon \sum s_{j}\right)
\end{aligned}
$$


where $C>0$ is independent of $\left(s_{1}, \ldots, s_{s}\right)$. As the $G$-invariant measure on $X$ is given by a constant multiple of

$$
\int_{K} \int_{B^{+}} f(k b H) \prod \sinh \left(b^{\alpha}\right)^{p_{\alpha}} \cosh \left(b^{\alpha}\right)^{q_{\alpha}} d b d k
$$

where $B^{+}:=\left\{b \in B \mid \forall \alpha \in \Delta^{+}\left(\mathfrak{g}_{0}, \mathfrak{b}_{0}\right): b^{\alpha}>1\right\}, \exp (h)^{\alpha}=e^{\alpha(h)}, h \in \mathfrak{b}_{0}$, $p_{\alpha}=\operatorname{dim}\left\{x \in \mathfrak{g}_{0 \alpha} \mid \vartheta \tau(x)=x\right\}$, and $q_{\alpha}=\operatorname{dim}\left\{x \in \mathfrak{g}_{0 \alpha} \mid \vartheta \tau(x)=-x\right\}$ (see [3, Theorem 2.6], the theorem follows.

As in $\S 3$, we let $w^{*}$ be the unique element in the Weyl group of $\Delta_{\mathfrak{k}}$ such that $w^{*} \Delta_{\mathfrak{k}}^{+}=-\Delta_{\mathfrak{k}}^{+}$. Then it follows easily that $w^{*} \mathfrak{a}=\mathfrak{a}$ and $w^{*} \Delta_{c}^{+}=-\Delta_{c}^{+}$. Let $\nu:=-w^{*}(\mu+\rho)=-w^{*} \mu+\rho_{c}-\rho_{n}$. Then relative to $\Delta^{+}, \nu$ is dominant regular and $\mu_{F}(\nu)=-w^{*} \mu$. We recall now the construction of the FlenstedJensen function to the parameter $\nu$. Let $\mathfrak{g}_{0}^{d}:=\mathfrak{h}_{0 k} \oplus i \mathfrak{h}_{0 p} \oplus i \mathfrak{q}_{0 k} \oplus \mathfrak{q}_{0 p}$ as before. Then $\mathfrak{g}_{0}^{d}$ is semisimple with Cartan-involution $\tau$ and "Iwasawa-a $a_{0}$ " $i \mathfrak{a}_{0}$. Furthermore

$$
\mathfrak{g}_{0}^{d}=\{x \in \mathfrak{g} \mid \theta \eta(x)=x\},
$$

where $\eta$ is the conjugation $x \mapsto \tau(\bar{x})$. Let $\mathfrak{k}_{0}^{d}:=\mathfrak{h}_{0 k} \oplus i \mathfrak{h}_{0 p}$. Let $G^{d}$ denote the analytic subgroup of $G_{c}$ corresponding to $\mathfrak{g}_{0}^{d}$. Then $G^{d}=G_{c}^{\theta \eta}$. let $K^{d}:=H_{c} \cap$ $G^{d}=\exp \left(\mathfrak{k}_{0}^{d}\right), A^{d}:=\exp \left(i \mathfrak{a}_{0}\right)$, and $N^{d}=\exp \left(\mathfrak{n}^{+} \cap \mathfrak{g}_{0}^{d}\right)$. Then $G^{d}=K^{d} A^{d} N^{d}$ is an Iwasawa decomposition of $G^{d}$. If $x \in G^{d}$ we write correspondingly $x=k^{d}(x) a^{d}(x) n^{d}(x)$. Now $B \subset G^{d}$ and thus we may write, for $b \in B$,

$$
b=k^{d}(b) a^{d}(b) n^{d}(b) .
$$

But by $[14, \S 2]$ we may also write

$$
b=h k_{H}(b) p
$$

with $h \in H_{c}$ unique modulo $H_{c} \cap K_{c}, k_{H}(b) \in K_{c}$ and $p \in P^{+}$. But by Theorem 2.4 in [14], $k_{H}(b) \in \exp \left(i \mathfrak{a}_{0}\right)=A^{d}$. By applying the conjugation $\theta \eta$ to (5.3) (or by using the proof of [14, Theorem 2.4]) it follows that $k_{H}(b)=$ $a^{d}(b), p=n^{d}(b)$, and $h=k^{d}(b)$ (modulo $K_{c} \cap H_{c}$ ). Now the FlenstedJensen function corresponding to $\nu$ is uniquely determined by the following two properties (see [3, p. 268]):

(1) $\psi_{\nu}$ is $K^{\#}$-finite of type $\delta$.

(2) $\psi_{\nu}\left(b H^{\#}\right)=\int_{K \cap H} a^{d}\left(b^{-1} k\right)^{-(\nu+\rho)} d k, b \in B$.

Theorem 5.4. Let $v \in \mathbf{V}$ be $K_{c}^{\# \tau}$-invariant and such that $\left\langle v, \nu^{0}\right\rangle=1$. Then $\Phi_{v}=\psi_{\nu}$. In particular $\mathbf{H}_{\delta} \cong \overline{\mathbf{F}}_{\nu}$.

Proof. Let $\nu^{0}$ and $v$ be as above. In the $K^{\#}$-variable, the function $\psi_{\nu}$ has an analytic continuation to a holomorphic function on $K_{c}^{\#}$ such that for $\kappa(x) \in$ $K_{c} \cap G^{d}$,

$$
\psi_{\nu}\left(x H^{\#}\right)=\int_{K \cap H} a^{d}\left(x^{-1} k\right)^{-(\nu+\rho)} d k=\left\langle v, \delta^{\vee}(x) \nu^{0}\right\rangle
$$


(see [3, pp. 268, 269]). Let $b^{-1} \in B, b^{-1}=k_{d} a_{d} n_{d}$. Then by the above, $n_{d} \in$ $P^{+} \cap G^{d}$. Thus $k^{-1} n_{d} k \in N^{d}$ for all $k \in K \cap H$. Hence $a^{d}\left(b^{-1} k\right)=a^{d}\left(a_{d} k\right)$ and

$$
\begin{aligned}
\psi_{\nu}\left(b H^{\#}\right) & =\int_{K \cap H} a^{d}\left(b^{-1} k\right)^{-(\nu+\rho)} d k \\
& =\int_{K \cap H} a^{d}\left(a_{d} k\right)^{-(\nu+\rho)} d k \\
& =\psi_{\nu}\left(a_{d}^{-1} H^{\#}\right)=\left\langle v, \delta^{\vee}\left(a_{d}\right)^{-1} \nu^{0}\right\rangle=\left\langle v, \delta^{\vee}\left(k_{H}\left(b^{-1}\right)^{-1}\right) \nu^{0}\right\rangle \\
& =\left\langle v, \delta^{\vee}\left(\gamma^{\#}(b)\right) \nu^{0}\right\rangle=\Phi_{v}(b) .
\end{aligned}
$$

Lemma 5.5. Assume that $\mathfrak{g}_{0}$ is simple. Let $\delta$ and $\mu$ be as in Theorem 5.3. Let $\gamma$ be the highest root in $\Delta_{n}^{+}$. Then

$$
\langle\nu+\rho, \lambda\rangle \leq\langle\mu+\rho, \gamma\rangle<0
$$

for all weights $\nu$ of $\delta$ and all $\lambda \in \Delta_{n}^{+}$.

Proof. First we notice that $\langle\gamma, \beta\rangle \geq 0$ for $\beta \in \Delta_{c}^{+},(\gamma+\beta$ is never a root $)$. Furthermore if $w \in W\left(\Delta_{c}^{+}\right)$and $\lambda \in \Delta_{n}^{+}$then $w \lambda \in \Delta_{n}^{+}$and there is a sum $Q$ of positive compact roots such that $w \lambda=\gamma-Q$. Let $\lambda$ and $\nu$ be as in the lemma. Choose $w \in W\left(\Delta_{c}^{+}\right)$such that $w(\nu+\rho)$ is $\Delta_{c}^{+}$-dominant. Then

$$
\begin{aligned}
\langle\nu+\rho, \lambda\rangle & =\langle w(\nu+\rho), w \lambda\rangle \\
& =\langle w(\nu+\rho), \gamma\rangle-\langle w(\gamma+\rho), Q\rangle \\
& \leq\langle w(\nu+\rho), \gamma\rangle
\end{aligned}
$$

as $w(\gamma+\rho)$ is $\Delta_{c}^{+}$-dominant. As $w \rho-\rho=-P_{1}$ and $w \nu=\mu-P$, where $P_{1}$ and $P$ are sums of positive compact roots, we now have

$$
\begin{aligned}
\langle w(\nu+\rho), \gamma\rangle & =\langle w \nu+\rho, \gamma\rangle+\langle w \rho-\rho, \gamma\rangle \\
& =\langle\mu+\rho, \gamma\rangle-\left\langle P+P_{1}, \gamma\right\rangle \\
& \leq\langle\mu+\rho, \gamma\rangle<0 . \quad \square
\end{aligned}
$$

6. AN EXAMPLE: THE HOLOMORPHIC DISCRETE SERIES OF $G$

We now specialize to the case $G \cong(G \times G) / d(G)$, where $d(G)$ is the diagonal $\{(x, x) \mid x \in G\}$. We will use the notation from the preceding sections. Let $\mathfrak{g}^{\sim}:=\mathfrak{g} \times \mathfrak{g}$ and define $\tau(X, Y):=(Y, X)$. Then $\mathfrak{h}=d(\mathfrak{g})=\{(X, X) \mid X \in \mathfrak{g}\}$ and $\mathfrak{q}=\{(X,-X) \mid X \in \mathfrak{g}\}$. Let $\mathfrak{c}_{\mathfrak{k}}$ be the center of $\mathfrak{k}$. That $G$ is of Hermitian type means that $\mathfrak{z}_{\mathfrak{g}}\left(\mathfrak{c}_{\mathfrak{p}}\right)=\mathfrak{k}$ or, equivalently, that $G / K$ is a bounded symmetric domain. Let $\mathfrak{c}:=\left\{(X,-X) \mid X \in \mathfrak{c}_{\mathrm{p}}\right\}$ and $\mathfrak{a}:=\{(X,-X) \mid X \in \mathfrak{t}\}$. Then we have the following identifications, where the superscript $\sim$ denotes the construction relative to $\mathfrak{g}^{\sim}$, and $\alpha^{\sim}=\frac{1}{2}(\alpha,-\alpha): \Delta=\left\{\alpha^{\sim} \mid \alpha \in \Delta_{\mathfrak{g}}\right\}=\Delta_{\mathfrak{g}}^{\sim}$, $\Delta^{+}=\left(\Delta_{\mathfrak{g}}^{+}\right)^{\sim}$,

$$
\mathfrak{g}_{\alpha}^{\sim}=\mathfrak{g}_{\alpha} \times \mathfrak{g}_{-\alpha}, \quad \tilde{p}^{\sim^{+}}=\mathfrak{p}^{+} \times \mathfrak{p}^{-}, \quad \tilde{p}^{\sim^{-}}=\mathfrak{p}^{-} \times \mathfrak{p}^{+} .
$$


Let $\delta^{\sim}$ be a finite-dimensional irreducible holomorphic representation of $K_{c}^{\sim}$ containing a nonzero $d\left(K_{c}\right)$-fixed vector. Then $\delta^{\sim} \cong \delta \otimes \delta^{\vee}$, where $\delta$ is an irreducible representation of $K_{c}$ and every $d\left(K_{c}\right)$-fixed vector $\nu^{0}$ for $\delta^{\nu^{\vee}}$ is given by a constant multiple of $\nu^{0}=\sum_{j=1}^{n} \nu_{j} \otimes v_{j}$ where $\left\{v_{1}, \ldots, v_{n}\right\}$ is a base of $\mathbf{V}$ and $\left\{\nu_{1}, \ldots, \nu_{n}\right\}$ is the dual base. Let $\mu$ be the highest weight of $\delta$; then the highest weight $\mu_{\delta^{\sim}}$ of $\delta^{\sim}$ is $2 \mu^{\sim}=(\mu,-\mu)$. As our " $\rho$-functional" relative to the above $\Delta^{+}$is now given by $\rho=2 \rho_{\mathfrak{g}}^{\sim}$, we have

$$
\left\langle\mu_{\delta^{\sim}}+\rho, \alpha^{\sim}\right\rangle=\left\langle\mu+\rho_{\mathfrak{g}}, \alpha\right\rangle
$$

for all $\alpha \in \Delta_{\mathfrak{g}}$. Hence $\left\langle\mu_{\delta^{\sim}}+\rho, \alpha^{\sim}\right\rangle<0$ for all $\alpha^{\sim} \in \Delta_{n}^{+}$if and only if $\left\langle\mu+\rho_{\mathfrak{g}}, \alpha\right\rangle<0$ for all $\alpha \in \Delta\left(\mathfrak{p}^{+}, \mathfrak{t}\right)$. Assume $\left\langle\mu+\rho_{\mathfrak{g}}, \alpha\right\rangle<0$ for all $\alpha \in \Delta\left(\mathfrak{p}^{+}, \mathfrak{t}\right)$. Let $\left(\varepsilon_{\delta^{\sim}}, \mathbf{H}_{\delta^{\sim}}\right)$ be the holomorphic discrete series representation of the last section. Then $\varepsilon_{\delta^{\sim}} \cong \pi \otimes \pi^{\vee}$, where $\pi$ is an irreducible unitary representation of $G$. As a representation of $G \cong G \times 1 \hookrightarrow G \times G \varepsilon_{\delta^{\sim}}$ is isomorphic to $\pi \otimes 1$, where 1 stands for the identity on $\mathbf{V}_{\pi}$. Let $x \in G$. Then

$$
\begin{aligned}
\left(x^{-1}, 1\right) & =\left(p^{-}(x)^{-1} k_{c}(x)^{-1} p^{+}(x)^{-1}, 1\right) \\
& =\left(p^{-}(x), p^{-}(x)\right)^{-1}\left(k_{c}(x)^{-1}, 1\right)\left(p^{+}(x)^{-1}, p^{-}(x)\right) .
\end{aligned}
$$

Hence $\gamma(x)=\left(k_{c}(x), 1\right) \bmod d\left(K_{c}\right)$. Choose the base $\left\{\nu_{1}, \ldots, \nu_{n}\right\}$ such that $\nu_{1}$ is a highest weight vector. Define $\Phi_{v}(x):=\Phi_{v \otimes \nu_{1}}((x, 1) d(G))$. Then

$$
\Phi_{v}(x)=\left\langle\delta\left(k_{c}(x)^{-1}\right) v, \nu_{1}\right\rangle
$$

and this is exactly the generating function for the holomorphic discrete series (see [10, p. 159]).

Theorem 6.1. Let $\delta^{\sim} \cong \delta \otimes \delta^{\vee}$ be a holomorphic representation of $K_{c}$. Let $\mu_{\delta^{\sim}}$ (resp. $\mu$ ) be the highest weight of $\delta^{\sim}$ (resp. $\delta$ ). Then

(1) $\left\langle\mu_{\delta^{\sim}}+\rho, \alpha^{\sim}\right\rangle<0$ for all $\alpha^{\sim} \in \Delta_{n}^{+}$if and only if $\left\langle\mu+\rho_{g}, \alpha\right\rangle<0$ for all $\alpha \in \Delta\left(\mathfrak{p}^{+}, \mathfrak{t}\right)$.

(2) Assume $\left\langle\mu_{\delta^{\sim}}+\rho, \alpha^{\sim}\right\rangle<0$ for all $\alpha^{\sim} \in \Delta_{n}^{+}$. Let $\varepsilon_{\delta^{\sim}}$ be the holomorphic discrete series representation of $G \times G / d(G)$ in $\mathbf{H}_{\delta^{\sim}}$, and let $\pi_{\delta}$ be the holomorphic discrete series representation of $G$ with lowest $K$-type $\delta$. Then $\varepsilon_{\delta^{\sim}} \cong \pi_{\delta} \otimes \pi_{\delta}^{\vee}$.

\section{The Realization of $\mathbf{H}_{\delta}$ as an analytic continuation of THE HOLOMORPHIC DISCRETE SERIES OF $\mathbf{G}^{\#}$}

In this section we recall the construction of a part of the analytic continuation of the holomorphic discrete series (see [22] or [16]) and use the results in $\S 1$ to determine the intertwining operators from the discrete series of $X^{\#}$. In particular it will follow that the multiplicity of $\varepsilon_{\delta}$ in $\mathbf{L}_{\xi}^{2}\left(X^{\#}\right)$ is one (see also [5] and [2]). We will always assume that $\delta$ and $\mu$ are as in $\S 5$; in particular, $\langle\mu+\rho, \alpha\rangle<0$ for all $\alpha \in \Delta_{n}^{+}$. 
Let $\mathscr{O}(\mathbf{V})$ be the space of $\mathbf{V}$-valued holomorphic functions on $D$. Via the Killing form we can identify $U\left(\mathfrak{p}^{-}\right) \otimes \mathbf{V}$ with the space of $\mathbf{V}$-valued polynomial functions on $\mathfrak{p}^{+}$and thus, by restriction, $U\left(\mathfrak{p}^{-}\right) \otimes \mathbf{V} \subset \mathscr{O}(\mathbf{V})$. For $u \in U(\mathfrak{g})$ define $u^{*}$ by $X^{*}=-\bar{X}, X \in \mathfrak{g}$. Define a sesquilinear form $(\cdot \mid \cdot)_{\delta}$ on $U\left(\mathfrak{p}^{-}\right) \otimes \mathbf{V}$ by

$$
\left(u \otimes v \mid u_{1} \otimes v_{1}\right)_{\delta}:=\left(\delta\left(p_{\mathfrak{k}}\left(u_{1}^{*} u\right)\right) v \mid v_{1}\right)_{V} .
$$

Then it can be shown (and this will follow from the proof of Theorem 7.2) that $(\cdot \mid \cdot)_{\delta}$ is positive definite. Let $\mathscr{O}_{\delta}$ denote the Hilbert space completion of $U\left(\mathfrak{p}^{-}\right) \otimes \mathbf{V}$. Then $\mathscr{O}_{\delta} \subset \mathscr{O}(\mathbf{V})$. Define a representation $\pi_{\delta}$ of $G^{\#}$ in $\mathscr{O}(\mathbf{V})$ by

$$
\left[\pi_{\delta}(x) f\right](z):=m(x, z) f\left(x^{-1} z\right),
$$

where $m(x, z):=\delta\left(k_{c}^{\#}\left(\kappa(x)^{-1} \exp (z)\right)^{-1}\right)$ and the lifting $k_{c}^{\#}$ is defined in the same manner as $\gamma^{\#}$. Then $\mathscr{O}_{\delta}$ is invariant under $\pi_{\delta}$.

Theorem 7.1 (H. Rossi, M. Vergne and N. Wallach). $\left(\pi_{\delta}, \mathscr{O}_{\delta}\right)$ is an irreducible unitary representation of $G^{\#}$ and $U\left(\mathfrak{p}^{-}\right) \otimes \mathbf{V}$ is the space of $K^{\#}$-finite vectors. If $\left\langle\mu+\rho_{\mathfrak{g}}, \alpha\right\rangle<0$ for all $\alpha \in \Delta\left(\mathfrak{p}^{+}, \mathfrak{t}\right)$, then $\pi_{\delta}$ is a holomorphic discrete series representation.

For $z=x \cdot 0 \in D$, we define $\Phi_{z}: X^{\#} \rightarrow \mathbf{V}$ by

$$
\Phi(z, y)=\Phi_{z}(y):=\delta\left(k_{c}^{\#}(x)\right) \Phi_{\delta^{v}}\left(x^{-1} y\right) .
$$

The function $\Phi(\cdot, y)$ is holomorphic and $\pi_{\delta}(x) \Phi(\cdot, y)=\Phi(\cdot, x y)$. For $f \in$ $\mathbf{L}_{\xi}^{2}\left(X^{\#}\right)$ set

$$
\begin{aligned}
T f(z) & :=\int_{X} f(x) \Phi_{z}(x) d x \\
& =\int_{X} \operatorname{pr}(f)(x) \Phi_{z}(x) d x
\end{aligned}
$$

where $\operatorname{pr}: \mathbf{L}_{\xi}^{2}\left(X^{\#}\right) \rightarrow \mathbf{H}_{\delta}$ is the orthogonal projection onto $\mathbf{H}_{\delta}$. Then $T f \in$ $\mathscr{O}(\mathbf{V})$ and

$$
\begin{aligned}
T\left(\varepsilon_{\delta}(y) f\right)(z) & =\int_{X} f\left(y^{-1} x\right) \Phi_{z}(x) d x \\
& =\int_{X} f(x) \Phi_{z}(y x) d x \\
& =\pi_{\delta}(y) T f(z) .
\end{aligned}
$$

Theorem 7.2. The representations $\varepsilon_{\delta}$ and $\pi_{\delta}$ are equivalent and

$$
\operatorname{Hom}_{G^{*}}\left(\mathbf{L}_{\xi}^{2}\left(X^{\#}\right), \mathscr{O}_{\delta}\right)=\mathbb{C} T,
$$

where $T$ is defined as above. In particular the multiplicity of $\pi_{\delta}$ in $\mathbf{L}_{\xi}^{2}\left(X^{\#}\right)$ is one.

Proof. The lowest $K^{\#}$-type of $\pi_{\delta}$ are just the constants $z \rightarrow u, u \in \mathbf{V}$. Thusby using the same idea as in $\S 2$ and Lemma 1.1 -it follows easily that pointevaluation in $\mathscr{O}_{\delta}$ is continuous. Let $U: \mathbf{L}_{\xi}^{2}\left(X^{\#}\right) \rightarrow \mathscr{O}_{\delta}$ be a nonzero unitary 
intertwining operator and define $\psi, \psi_{z} \in \mathbf{L}_{\xi}^{2}\left(X^{\#}, \mathbf{V}\right)$ by $\psi_{z}:=\left({ }^{t} U \otimes 1\right)\left(\mathscr{K}_{z}\right)$ and $\psi=\psi_{0}$ as in $\S 1$; i.e.

$$
U f(z)=\int_{X} f(x) \psi_{z}(x) d x .
$$

Then $\psi_{y 0}(x)=\delta\left(k_{c}(y)\right) \psi\left(y^{-1} x\right)$ by Lemma 1.4. And as in [14],

$$
z \mapsto \psi_{z}(x) \text { is holomorphic for all } x \in X^{\#} \text {. }
$$

As $\psi\left(k H^{\#}\right)=\delta(k) \psi\left(1 H^{\#}\right)$ it follows that $\psi\left(1 H^{\#}\right)=: u^{0}$ is a nonzero $K^{\# \tau}$ fixed vector in $\mathbf{v}$. Define $\Phi_{\delta^{\vee}}$ by using $u^{0}$, i.e. $\Phi_{\delta^{v}}\left(1 H^{\#}\right)=u^{0}$, and define $\Phi(z, y)$ as above using this $\Phi_{\delta^{v}}$. Then it follows that

$$
\psi_{h \cdot z}\left(1 H_{c}^{*}\right)=\Phi_{h \cdot z}\left(1 H_{c}^{\#}\right)=\delta\left(k_{c}^{\#}(h)\right) u^{0}
$$

for all $h \in H^{\#}$. As $D$ can be realized as a simply-connected open subset in $H_{c}^{\#} / H_{c}^{\#} \cap K_{c}^{\#}$, it follows from (7.3) that $\psi_{z}\left(1 H^{\#}\right)=\Phi_{z}\left(1 H^{\#}\right)$ for all $z \in D$. Thus $\psi_{z}=\Phi_{z}$ and $U=T$.

That those representations are equivalent can be seen from the parameters determining them. Another proof is to define $\Phi_{z}$ and $T$ as above and then show that $T \mid \mathbf{H}_{\delta}$ (simply denoted by $T$ ) is a nonzero intertwining operator. Let $A(z) \in \operatorname{End}(\mathbf{V})$ be defined by

$$
A(z) u:=T\left(\Phi_{u}\right)(z)=\delta\left(k_{c}^{\#}(y)\right) \int \Phi_{u}(x) \Phi_{\delta^{v}}\left(y^{-1} x\right) d x, \quad z=y 0 .
$$

Write $\kappa(y)=p^{+}(y) k_{c}(y) p^{-}(y)$ and use Theorem 5.1, parts (1) and (6), to show that $A(y)=A(1)$. Furthermore $A(1)(\delta(k) u)=\delta(k) A(1) u$. Hence there is a $c(\mu) \in \mathbb{C}$ such that $A(1)=c(\mu)$ id. Identifying $\mathbf{V}$ and $\mathbf{V}^{*}$ as usual, $\overline{\Phi_{w}(x)}=$ $\left(\Phi_{\delta^{\vee}}(x) \mid w\right)$. Hence $\left|\Phi_{w}\right|^{2}=\int \Phi_{w}(x) \overline{\Phi_{w}(x)} d x=(A(0) w \mid w)=c(\mu)|w|^{2}$ and $c(\mu)>0$ and $T\left(\mathbf{H}_{\delta}(\delta)\right)$ is the space of constants $z \mapsto u$. For $u, u_{1} \in U\left(\mathfrak{p}^{-}\right)$ we have, furthermore,

$$
\begin{array}{rlrl}
\left(u \Phi_{v} \mid u_{1} \Phi_{w}\right) & =\left(u_{1}^{*} u \Phi_{v} \mid \Phi_{w}\right) & & \text { as } \mathbf{H}_{\delta} \text { is unitary } \\
& =\left(q_{\mathfrak{k}}\left(u_{1}^{*} u\right) \Phi_{v} \mid \Phi_{w}\right) & & \text { by Theorem 5.2(5) } \\
& =\left(\Phi_{q_{\mathfrak{k}}\left(u_{\mathrm{i}}^{*} u\right) v} \mid \Phi_{w}\right) & \\
& =c(\mu)\left(q_{\mathfrak{k}}\left(w^{*} u\right) v \mid w\right) & \\
& =c(\mu)\left(u \otimes v \mid u_{1} \otimes w\right)_{\delta} . &
\end{array}
$$

Thus $c(\mu)^{-1 / 2} T$ is unitary-and, in particular, continuous. Now the theorem follows.

If $\mu+\rho_{\mathfrak{g}}$ is $\Delta\left(\mathfrak{p}^{-}, \mathfrak{t}\right)$-dominant, then there is a constant $d(\mu)>0$ such that

$$
\mathscr{O}(\mu) \ni f \mapsto F_{f}: x \mapsto d(\mu) \delta\left(k_{c}^{\#}(x)\right)^{-1} f(x z) \in \mathbf{H}_{2}\left(\mu+\rho_{\mathfrak{g}}\right)
$$

is a unitary $G^{\#}$-isomorphism (see [15, p. 23]). In this realization, the formula for the intertwining operator is given by the function $\Phi_{\delta^{v}}$. Theorem 3.2 and 


\section{Theorem 4.2 now read}

Theorem 7.3. Assume that $\mu+\rho_{\mathfrak{g}}$ is $\Delta^{+}\left(\mathfrak{p}^{-}, \mathfrak{t}\right)$-dominant. Then every continuous intertwining operator $T: \mathbf{H}_{\delta} \rightarrow \mathbf{H}_{2}\left(\mu+\rho_{\mathfrak{g}}\right)$ is given by

$$
T \phi(y)=\int_{X} \phi(x) \delta\left(\gamma^{\#}\left(y^{-1} x\right)\right) v d x
$$

where $v$ is a $K_{c}^{\# \tau}$-invaiant vector in $\mathbf{V}$. If $\pi_{\mu+\rho_{\mathrm{g}}}$ is integrable, then up to a constant

$$
T^{-1} f(x)=\int_{D}\left(f(y) \mid \delta\left(\gamma^{\#}\left(y^{-1} x\right)\right) v\right)_{V} d y
$$

\section{REFERENCES}

1. E. P. van den Ban, Asymptotic behaviour of matrix coefficients related to reductive symmetric spaces, Proc. Koninklijke Nederl. Akad. Wetensch. A 90 (1987).

2. F. Bien, Multiplicity one for exceptional symmetric spaces, Preprint, May 1987; Spherical $\mathscr{D}$-modules and representations of reductive Lie groups, Ph.D. Thesis, M.I.T., June 1986.

3. M. Flensted-Jensen, Discrete series for semisimple symmetric spaces, Ann. of Math. 111 (1980), 253-311.

4. __ $K$ - -finite joint eigenfunctions of $U(\mathfrak{g})^{K}$ on a non-Riemannian semisimple symmetric space $G / H$, Lecture Notes in Math., vol. 880, Springer-Verlag, Berlin, 1981.

5. __ Analysis on non-Riemannian symmetric spaces, CBMS Regional Conf. Ser. in Math., no. 61, Amer. Math. Soc., Providence, R.I., 1987.

6. E. Gutkin, Invariant elliptic operators and unitary representations, Ph.D. Thesis, Brandeis Univ., 1978; Preprint, 1979.

7. H. Hecht and W. Schmid, On integrable representations of semisimple Lie groups, Math. Ann. 220 (1976), 147-150.

8. S. Helgason, Differential geometry, Lie groups and symmetric spaces, Academic Press, New York and London, 1978.

9. R. Hotta, On realization of the discrete series for semisimple Lie groups, J. Math. Soc. Japan 23 (1971), 384-407.

10. A. W. Knapp, Representation theory of semisimple groups: An overview based on examples, Princeton Univ. Press, Princeton, N. J., 1986.

11. M. Matsumoto, Discrete series for an affine symmetric space, Hiroshima Math. J. 11 (1981), 53-79.

12. T. Matsuki and T. Oshima, A description of discrete series for semisimple symmetric spaces, Adv. Stud. Pure Math., vol. 4, 1984, pp. 229-390.

13. G. Olafsson, Die Langlands Parameter für die Flensted-Jensensche fundamentale Reihe, Math. Scand. 55 (1984), 229-244.

14. G. Olafsson and B. Ørsted, The holomorphic discrete series for affine symmetric spaces. I, Mathematica Gottingensis, no. 43, 1987; J. Funct. Anal. 82 (1988), 126-159.

15. Imbedding of the discrete series of $G$ into $\mathbf{L}^{2}(X)$, Mathematica Gottingensis, no. 5, 1988.

16. H. Rossi and M. Vergne, Analytic continuation of the holomorphic discrete series of a semisimple Lie group, Acta Math. 136 (1976), 1-59.

17. H. Schlichtkrull, On some series of representations related to symmetric spaces, Mém. Soc. Math. France (N.S.), no. 15, 1984, pp. 277-289.

18. __ The Langlands parameters of Flensted-Jensen's discrete series for semisimple symmetric spaces, J. Funct. Anal. 50 (1983), 133-150. 
19. _ـ Hyperfunctions and harmonic analysis on symmetric spaces, Birkhäuser, Boston, Mass. and Basel, 1984.

20. P. C. Trombi and V. S. Varadarajan, Asymptotic behavior of eigenfunctions on a semisimple Lie groups: The discrete spectrum, Acta Math. 129 (1972), 237-280.

21. V. S. Varadarajan, Harmonic analysis on real reductive groups, Lecture Notes in Math., vol. 576, Springer-Verlag, Berlin, 1977.

22. N. Wallach, The analytic continuations of the discrete series. I, II, Trans. Amer. Math. Soc. 251 (1979), 1-17 and 19-37.

23. N. R. Wallach and J. A. Wolf, Completeness of Poincaré series for automorphic forms associated to the integrable discrete series, P. C. Trombi (ed.), Representation Theory of Reductive Groups, (Proc. Univ. of Utah Conf., 1982), Birkhäuser, Boston, Mass. and Basel, 1983, pp. 265-281.

24. G. Warner, Harmonic analysis on semi-simple Lie groups. I, Springer-Verlag, Berlin, 1972.

Mathematisches Institut der Universität Göttingen, Bunsenstrasse 3/5, D-3400 Göttingen, Deutschland

Matematisk Institut, Odense Universitet, Campusvej 55, DK-5230 Odense M, Danmark 